\title{
Membrane and luminal proteins reach the apicoplast by different trafficking pathways in the malaria parasite Plasmodium falciparum
}

\author{
Rahul Chaudhari ${ }^{1}$, Vishakha Dey ${ }^{2}$, Aishwarya Narayan ${ }^{2}$, Shobhona Sharma ${ }^{1}$, Swati Patankar Corresp. ${ }^{2}$ \\ 1 Department of Biological Sciences, Tata Institute of Fundamental Research, Mumbai, Maharashtra, India \\ 2 Department of Biosciences and Bioengineering, Indian Institute of Technology, Bombay, Mumbai, Maharashtra, India \\ Corresponding Author: Swati Patankar \\ Email address: patankar@iitb.ac.in
}

The secretory pathway in Plasmodium falciparum has evolved to transport proteins to the host cell membrane and to an endosymbiotic organelle, the apicoplast. The latter can occur via the ER or the ER-Golgi route. Here, we study these three routes using proteins Erythrocyte Membrane Protein-1 (PfEMP1), Acyl Carrier Protein (ACP) and glutathione peroxidase-like thioredoxin peroxidase $\left(\mathrm{PfTPx}_{\mathrm{GI}}\right)$ and inhibitors of vesicular transport. As expected, the $\mathrm{G}$ protein dependent vesicular fusion inhibitor $\mathrm{AlF}_{4}{ }^{-}$and microtubule destabilizing drug vinblastine block the trafficking of PfEMP-1, a protein secreted to the host cell membrane. However, while both PfTPx $_{G I}$ and ACP are targeted to the apicoplast, only ACP trafficking remains unaffected by these treatments. This implies that G-protein dependent vesicles do not play a role in classical apicoplast protein targeting. Unlike the soluble protein $A C P$, we show that PfTPx $_{G I}$ is localized to the outermost membrane of the apicoplast. Thus, the parasite apicoplast acquires proteins via two different pathways: first, the vesicular trafficking pathway appears to handle not only secretory proteins, but an apicoplast membrane protein, $\mathrm{PfTPx}_{\mathrm{Gl}}$. Second, trafficking of apicoplast luminal proteins appear to be independent of G-protein coupled vesicles. 
1 Membrane and luminal proteins reach the apicoplast by different trafficking pathways in

2 the malaria parasite Plasmodium falciparum

3 Rahul Chaudhari ${ }^{1}$, Vishakha Dey ${ }^{2}$, Aishwarya Narayan ${ }^{2}$, Shobhona Sharma ${ }^{1}$, Swati Patankar ${ }^{2}$

$4{ }^{1}$ Department of Biological Sciences, Tata Institute of Fundamental Research, Colaba, Mumbai

5400005 , India

$6{ }^{2}$ Department of Biosciences \& Bioengineering, Indian Institute of Technology Bombay, Powai,

7 Mumbai 400076, India

8 Corresponding author:

9 Swati Patankar ${ }^{2}$

10 Email addresses:patankar@iitb.ac.in 


\section{$\underline{\text { Abstract }}$} host cell membrane and to an endosymbiotic organelle, the apicoplast. The latter can occur via the ER or the ER-Golgi route. Here, we study these three routes using proteins Erythrocyte Membrane Protein-1 (PfEMP1), Acyl Carrier Protein (ACP) and glutathione peroxidase-like thioredoxin peroxidase $\left(\mathrm{PfTPx}_{\mathrm{Gl}}\right)$ and inhibitors of vesicular transport. As expected, the $\mathrm{G}$ protein dependent vesicular fusion inhibitor $\mathrm{AlF}_{4}^{-}$and microtubule destabilizing drug vinblastine block the trafficking of PfEMP-1, a protein secreted to the host cell membrane. However, while both $\mathrm{PfTPx}_{\mathrm{Gl}}$ and $\mathrm{ACP}$ are targeted to the apicoplast, only ACP trafficking remains unaffected by these treatments. This implies that G-protein dependent vesicles do not play a role in classical apicoplast protein targeting. Unlike the soluble protein $\mathrm{ACP}$, we show that $\mathrm{PfTPx}_{\mathrm{Gl}}$ is localized to the outermost membrane of the apicoplast. Thus, the parasite apicoplast acquires proteins via two different pathways: first, the vesicular trafficking pathway appears to handle not only secretory proteins, but an apicoplast membrane protein, $\mathrm{PfTPx}_{\mathrm{Gl}}$. Second, trafficking of apicoplast luminal proteins appear to be independent of G-protein coupled vesicles.

\section{$\underline{\text { Introduction }}$}

Plasmodium falciparum parasites export proteins to the plasma membrane of host erythrocytes, cells that do not possess their own trafficking machinery. In order to do so, the parasite extensively modifies the host cell to make a favorable niche for survival (Moxon et al. 2011). The parasite can, therefore, be considered a major secretory cell.

In the secretory pathway, proteins are targeted to their destinations by the endomembrane system, starting with the proteins' entry into the endoplasmic reticulum (ER), a process facilitated by $\mathrm{N}$-terminal signal sequences that are usually hydrophobic in nature. From the ER, proteins are sent to the Golgi and further to their final destinations. In P. falciparum, the ER consists of a tubular, interconnected network that surrounds the nucleus, while the unstacked Golgi apparatus consists of distinct cis- and trans- compartments (Struck et al. 2005; van Dooren et al. 2005). In mammalian cells, the position and integrity of the ER and Golgi are maintained 
52 by microtubules, which also act as tracks for vesicles that target proteins via the secretory 53 pathway (Cole \& Lippincott-Schwartz 1995).

Once inside the ER, the proteins navigate different paths according to their targeting signals and destinations (Deponte et al. 2012). For example,P. falciparum Erythrocyte

Membrane Protein-1 (PfEMP-1) has N-terminal transmembrane regions which act as signal sequences,sending the protein via the secretory route to the parasite plasma membrane from where they are exported to the host cell surface (Knuepfer et al. 2005). In addition to export, proteins are also trafficked internally to parasite subcellular compartments, including an unusual relict plastid, the apicoplast. The apicoplast is believed to be acquired by secondary endosymbiosis and is surrounded by four lipid bilayers (Lemgruber et al. 2013; McFadden \& Roos 1999). The organelle possesses a $35 \mathrm{~kb}$ circular genome that codes for a handful of housekeeping genes and as a result, is heavily dependent on the import of nuclear-encoded proteins (Marechal \& Cesbron-Delauw 2001).

A protein destined for the apicoplast lumen is marked by an N-terminal bipartite signal, comprising of a signal peptide, for entry into the secretory pathway at the ER, and a transit peptide, required for luminal import by translocons upon reaching the apicoplast (Tonkin et al. 2006b; Waller et al. 2000). Once inside, the transit sequence is removed by an organellar peptidase to form a mature functional protein (van Dooren et al. 2002).

Since proteins that enter the ER usually follow the secretory route, the trafficking of a luminal protein from the ER to the apicoplast might be expected to go via the Golgi. However, in $P$. falciparum, there are two models for trafficking of apicoplast proteins from the ER lumen. In one report, it is suggested thata luminal protein Acyl Carrier Protein fused to Green Fluorescent Protein (ACP-GFP) is transferred from the ER to the apicoplast bypassing the Golgi(Tonkin et al. 2006b). This has been hypothesized to occur directly via vesicles, or due to transient contacts between the membranes of the two organelles. Interestingly, in another study ACP-GFP was suggested to transit through the Golgi(Heiny et al. 2014); reconciling these two reports remains an open area.

Another apicoplast protein, the glutathione peroxidase-like thioredoxin peroxidase $\left(\mathrm{PfTPx}_{\mathrm{Gl}}\right)$ of P. falciparum localizes to the apicoplast and/or mitochondrion. This heterogeneous localization of $\mathrm{PfTPx}_{\mathrm{G} 1}$ is completely disrupted upon BFA treatment suggesting an ER-Golgi route for organellar localization(Chaudhari et al. 2012). In contrast to ACP, its targeting does not 
83 involve the cleavage of $\mathrm{N}$-terminal signal sequences. Another group has localized this protein to 84 the apicoplast and the cytosol by fusion of N-terminal 47 amino acids to GFP(Kehr et al. 2010).

Clearly, in P. falciparum, once proteins enter the ER, they could have different fates. These include export via the Golgi and secretory pathway, trafficking to the apicoplast via the Golgi and trafficking to the apicoplast directly from the ER. In this report, we study the trafficking of twoapicoplast proteins $\left(\mathrm{PfTPx}_{\mathrm{Gl}}\right.$ and $\left.\mathrm{ACP}\right)$. Inhibition of vesicular fusion and transport were carried out using aluminum tetrafluoride $\left(\mathrm{AlF}_{4}^{-}\right)$, a small molecule inhibitor of vesicle fusion to target membranes and vinblastine, a microtubule depolymerizing agent that disrupts vesicular transport. These inhibitors have been well characterized in Plasmodium falciparum and shown to target the same functions as in other eukaryotes (Chakrabarti et al. 2013; Taraschi et al. 2001). $\mathrm{PfTPx}_{\mathrm{Gl}}$ localization is disrupted by $\mathrm{AlF}_{4}{ }^{-}$and vinblastine while the localization of luminal apicoplast proteins (including ACP) is unaffected by the same concentrations of these compounds, suggesting that $\mathrm{PfTPx}_{\mathrm{Gl}}$ and ACP trafficking proceeds by two different routes. The nature of the signals on these proteins and the signals on different types of vesicles that dictate the choice of the trafficking routes emanating from the ER is now an avenue for future research. One such signal to direct apicoplast proteins through the Golgi could be membrane localization: here we show that $\operatorname{PfTPx}_{\mathrm{Gl}}$ is associated with the outermost membrane of apicoplasts, suggesting that, unlike luminal proteins, the protein is trafficked on vesicular membranes.

\section{$\underline{\text { Materials \& Methods }}$}

\section{$\underline{\text { Ethical Clearance }}$}

The work was approved by the Institute ethics committee and Institute biosafety committee at Indian Institute of Technology Bombay. Written informed consent was provided by all the blood donors.

\section{$\underline{\text { In vitro culture of } P \text {. falciparum erythrocytic stages }}$}

For parasite culture, blood was collected from healthy donors who provided written informed consent. P. falciparum 3D7 strain was cultured in RPMI 1640 [Gibco ${ }^{\circledR}$ ] with an additional $2 \mathrm{mg} \mathrm{ml}^{-1}$ sodium bicarbonate $\left(\operatorname{Sigma}^{\circledR}\right)$, supplemented with 10\% B+ human plasma,

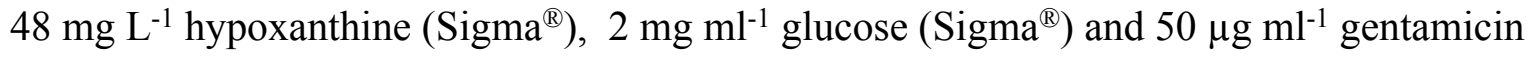


112 (Abbott). A hematocrit of 3\% was maintained using human $\mathrm{B}^{+}$red blood cells. Parasites were

113 tightly synchronized for all experiments except for the Western blot experiment showing 114 membrane extraction (Figure 6A). Briefly, the parasites were synchronized at the early rings

115 stage (4-5 hours post infection) by treatment with 5\% sorbitol (10 volumes of the RBC pellet) for

11610 minutes at $37^{\circ} \mathrm{C}$ followed by two washes with incomplete RPMI. Resulting pellet was

117 suspended in complete medium as described above. The treatment was repeated after 4 hours to

118 synchronize the cultures tightly. The synchronization was confirmed by observing smears

119 prepared from the synchronized cultures.

120 Immunofluorescence microscopy

121 Immunofluorescence microscopy of $P$. falciparum D10-ACP leader-GFP parasites was

122 done as described earlier with slight modifications (Tonkin et al. 2004). All steps up to

123 incubation with secondary antibodies were performed according to Tonkin et al. The details

124 about combinations and dilutions of the antibodies and their method of generation and checking

125 the specificity are shown in Table S1. Primary antibodies treatment was performed overnight at

$1264^{\circ} \mathrm{C}$ for all the proteins and secondary antibodies treatment was performed for 1.5 hours at room

127 temperature.

After incubation with secondary antibodies, the cells were subjected to three PBS

129 washes in suspension and allowed to settle on poly-L-lysine ( Sigma $\left.^{\circledR}\right)$ coated cover slips for 10

130 minutes. Cover slips were washed thrice in PBS, air dried and mounted using $0.1 \mathrm{mg} \mathrm{ml}^{-1} 1$,4-

131 diazylbicyclo[2.2.2] octane (DABCO, Sigma $\left.{ }^{\circledR}\right)$ on to glass slides. Slides were examined with

132 Olympus ${ }^{\circledR}$ FluoView ${ }^{\circledR 500}$ Confocal Laser Scanning Microscope. For each experiment

133 consisting of a control and treated cultures, all images were acquired at identical settings (laser

134 power ranging from $0.5-4 \%$ ). For clarity, the images were processed later for brightness and

135 contrast using ImageJ 1.46r where adjustments were applied to whole image. No non-linear

136 adjustments were performed.

137

138 Treatment of Plasmodium falciparum with inhibitors of vesicular trafficking

139 Small molecule inhibitors were used to disrupt the vesicular trafficking pathway. Small

140 molecules can have pleiotropic effects; therefore, drugs that affect different steps of vesicular

141 transport were chosen with the expectation of obtaining consistent results with all the treatments. 
142 Further, the same treatments are tested for their effects on three different proteins, in an attempt

143 to dissect out the pathways used by these proteins under the identical conditions.

144 For all drug treatments, it was important to ensure that the observed signal was not from

145 previously accumulated organellar protein. This would require treating the parasites with drugs

146 for a period of time that would be close to or greater than the half-life of the protein being

147 studied. The most stable protein studied here is likely to be GFP, whose half-life is estimated to

148 be 26 hours in mammalian cells (Corish \& Tyler-Smith 1999) however, can be as short as 2

149 hours in some cells (Halter et al. 2007). Hence, rather than treating parasites with high

150 concentrations of drugs for a few hours, as has been done previously (Kaderi Kibria et al. 2015;

151 Taraschi et al. 2001), we first determined the $\mathrm{IC}_{50}$ concentrations of the drugs and then treated

152 parasites for 18-20 hours with these lower concentrations(Fig. S1).

\section{$153 \underline{\text { I. IC }} \underline{50}_{\underline{0}}$ determination for the $\mathrm{AlF}_{4}$ treatment}

$154 \mathrm{IC}_{50}$ determination for the $\mathrm{AlF}_{4}$ - treated parasites were performed in 24 well plates where the

155 reduction in parasitemia at different $\mathrm{AlF}_{4}^{-}$concentrations was monitored. The experimental set-

156 up included $2 \mathrm{ml}$ of tightly synchronised $P$. falciparum 3D7 cultures grown as described

157 previously in the materials and methods section. For these experiments, two plates (two

158 biological replicates) were maintained containing two technical replicates for each $\mathrm{AlF}_{4}{ }^{-}$

159 concentration. For generation of $\mathrm{AlF}_{4}^{-}(1 \mu \mathrm{M}$ to $10 \mu \mathrm{M})$, appropriate amounts of $\mathrm{AlCl}_{3}\left(\operatorname{Sigma}^{\circledR}\right)$

160 (10mM stock) and $\mathrm{NaF}\left(\right.$ Sigma $\left.^{\circledR}\right)(1 \mathrm{M}$ stock) were added. The plates, kept in duplicate, were

161 maintained for two life cycles (96 hours). Spent media was replaced with fresh media every 24

162 hours with the addition of fresh $\mathrm{AlCl}_{3}$ and $\mathrm{NaF}$ for the generation of $\mathrm{AlF}_{4}^{-}$. Reduction in the

163 parasitemia at different concentrations of $\mathrm{AlF}_{4}^{-}$was assessed with smears prepared using Field's

164 stain every 24 hours. Percentage parasitemia as compared to controls were calculated by

165 subtracting the parasitemia of the $\mathrm{AlF}_{4}^{-}$treated cultures from the parasitemia of the control

166 (without $\mathrm{AlF}_{4}^{-}$) for each time point. The graph of percent parasitemia compared to the controls

167 was plotted against drug concentrations and 50\% inhibitory concentration $\left(\mathrm{IC}_{50}\right)$ was calculated

168 with calculated with non-linear regression of the sigmoidal dose response equation from

169 OriginPro for Windows.

170

171 II. Aluminum tetrafluoride treatment 
173

174

175

176

177

178

179 180

181 concentration). Briefly, for generation of $\mathrm{AlF}_{4}^{-}, \mathrm{AlCl}_{3}\left(\operatorname{Sigma}^{\circledR}\right)$ and $\mathrm{NaF}\left(\mathrm{Sigma}^{\circledR}\right)$ were combined in $5 \mathrm{ml}$ complete medium to a final concentration of $1.2 \mu \mathrm{M} \mathrm{AlCl}_{3}$ and $0.36 \mathrm{mM} \mathrm{NaF}$ $\left(\mathrm{AlCl}_{3}-10 \mathrm{mM}\right.$ stock and NaF - 1M stock). $150 \mu \mathrm{l}$ of packed infected red blood cells (having parasitemia of $5 \%$ early rings) were then added to $1.2 \mu \mathrm{M} \mathrm{AlF}_{4}{ }^{-}$containing complete medium (final hematocrit of $3 \%$ ). The cultures were incubated at $37^{\circ} \mathrm{C}$ for $18 \pm 2$ hours. The $\mathrm{AlF}_{4}^{-}$treated cultures were then washed three times with incomplete medium and a final wash with PBS. This was followed by preparation of immunofluorescence slides as described previously.

\section{Treatment of the $P$. falciparum D10-ACP ${ }_{\text {leader-GFP parasites with microtubule }}$}

destabilizing drugs

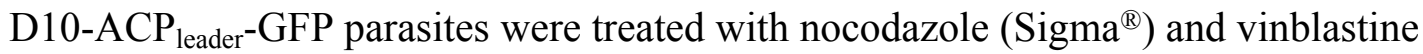
$\left(\right.$ Sigma $\left.^{\circledR}\right)$ at their $\mathrm{IC}_{50}$ concentrations $17 \mu \mathrm{M}$ and $100 \mathrm{nM}$ respectively in a final volume of $5 \mathrm{ml}$. Vinblastine stock of $50 \mu \mathrm{M}$ was prepared in phosphate buffered saline while a $10 \mathrm{mM}$ nocodazole stock solution was prepared in DMSO. Both stock solutions were sterilized by passing through $0.2 \mu$ membrane PVDF filters (Merck-Millipore). One culture flask was treated with an equal volume of DMSO (Sigma $\left.{ }^{\circledR}\right)$ as a control.

After $18 \pm 2$ hours incubation with drugs, $2 \mathrm{ml}$ of cultures were removed, washed three times with incomplete medium and a final wash with PBS and usedfor PfTPx $\mathrm{X}_{\mathrm{Gl}}$ and microtubule staining. This was followed by preparation of immunofluorescence slides as described previously. For assessing the reversion of localization in drug washed out parasites, the remaining cultures were washed thrice with complete medium and resuspended in a complete medium without drugs and subjected to additional incubation of 4 hours followed by preparation of immunofluorescence slides.

\section{Immunofluorescence microscopy of intact organelles}

For detection of membrane localized PfTPx ${ }_{\mathrm{Gl}}$, intact organelles were isolated according to a previous report(Mullin et al. 2006) except the parasites were lysed by expulsion through a 26-gauge needle (20 times) and the organelles including the apicoplast in the post-nuclear fraction were then centrifuged at $13000 \mathrm{~g}$ for 20 minutes, $4^{\circ} \mathrm{C}$. The organellar pellet was divided into two fractions. 
To detect proteins located on the membranes of organelles, intact organelles were re-

204

205

206

207

208

209

210

211

212

213

214

215

216

217

218

219

220

221

222

223

224

225

226

227

228

229

230

231

232

233

suspended in $1 \mathrm{X}$ assay buffer containing $1 \% \mathrm{BSA}$ and shaken at $4{ }^{\circ} \mathrm{C}$ for 30 minutes for blocking. This was followed by incubation with anti-PfTPx $\mathrm{Gl}_{\mathrm{Gl}}$ antibodies (1:100) and anti-GFP antibodies (1:250) for 4 hours at $4{ }^{\circ} \mathrm{C}$ in separate reaction tubes. After one wash with $1 \mathrm{X}$ assay buffer containing 1\% BSA, organelles were treated with secondary antibodies [goat anti-rabbit IgG $(\mathrm{H}+\mathrm{L})$ Alexa Fluor ${ }^{\circledR} 568$ (Invitrogen ${ }^{\mathrm{TM}}$ ) diluted 1:200 for detection of anti-PfTPxG1 antibodies and Goat anti-Mouse IgG $(\mathrm{H}+\mathrm{L})$ Alexa Fluor ${ }^{\circledR} 568$ (Invitrogen $\left.^{\mathrm{TM}}\right)$ (1:250) for detection of anti-GFP antibodies] in $1 \mathrm{X}$ assay buffer containing $1 \% \mathrm{BSA}$ for 1 hour at $4^{\circ} \mathrm{C}$. This was followed by a final wash with $1 \mathrm{X}$ assay buffer without $1 \%$ BSA and fixation with $4 \%$ paraformaldehyde and $0.0075 \%$ glutaraldehyde for 30 minutes on ice. This prep was then treated with primary and secondary antibodies and the slides were prepared as described previously. Mitochondria in the isolated organelles were visualized by staining with Mitotracker Red CM$\mathrm{H}_{2} \mathrm{XRos}$ (Invitrogen ${ }^{\mathrm{TM}}$ ) at $50 \mathrm{nM}$ final concentration for 30 minutes at room temperature.

To detect proteins within the organelles, organelles were first fixed with $1 \mathrm{X}$ assay buffer containing 4\% paraformaldehyde and $0.0075 \%$ glutaraldehyde for 30 minutes on ice. Organelles were then permeabilized with $0.1 \%$ Triton X-100 in $1 \mathrm{X}$ assay buffer for 10 minutes on ice. The preparation was blocked with 3\% BSA, treated with primary and secondary antibodies and the slides were prepared as described above.

\section{Differential solubilization of PfTPx ${ }_{\text {GI }}$ and Western blotting}

Approximately $6 \times 10^{9}$ parasites were lysed hypotonically in de-ionized water containing protease inhibitors followed by three rounds of freeze-thaw cycles. The resulting suspension was divided equally in three different parts. This was followed by centrifugation at $36,000 \mathrm{~g}$ for 30 minutes at $4{ }^{\circ} \mathrm{C}$. The supernatant containing soluble proteins was removed and the pellets were subjected to $1 \%$ Triton X-100-PBS for 30 minutes at $4^{\circ} \mathrm{C}$. This was followed by centrifugation at $36,000 \mathrm{~g}$ for 30 minutes at $4^{\circ} \mathrm{C}$ to obtain insoluble fraction and supernatant containing integral membrane proteins. Proteins were then quantified by bicinchoninic assay using BSA as a standard. $200 \mu \mathrm{g}$ of each protein fraction (soluble proteins and integral membrane proteins extracted with Triton X-100) was then separated on 15\% SDS-PAGE.

Proteins were transferred to polyvinylidenedifluoride (PVDF) membranes [pore size 0.45 $\mu \mathrm{m}$, Millipore $\left.{ }^{\mathrm{TM}}\right]$. The membranes were blocked for an hour with 3\% BSA/PBS. The membranes 
234 were then incubated for 3 hours in $0.5 \%$ Tween-20 $\left(\right.$ Sigma $\left.^{\circledR}\right) /$ PBS containing rabbit raised anti-

$235 \operatorname{PfTPx}_{\mathrm{Gl}}$ serum at 1:2000 dilution and mouse anti-GFP antibodies (for detection of ACP-GFP) at

236 1:1000 dilution at room temperature. This was followed by three washes with PBS. The proteins

237 were probed with horseradish peroxidase-conjugated goat anti-rabbit secondary antibodies

238 (Merck Biosciences) (1:2000) for 1.5 hours. This was followed by three washes with PBS and

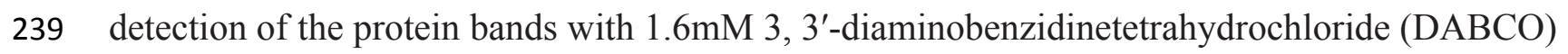

240 and $0.1 \%$ hydrogen peroxide as substrates in $10 \mathrm{ml}$ of $0.01 \mathrm{M}$ Tris ( $\mathrm{pH} 7.6)$. Molecular size of the

241 protein bands were determined with reference to pre-stained protein molecular weight markers

$242\left(\right.$ Fermentas $\left.^{\circledR}\right)$.

243

244

\section{Thermolysin treatment}

Thermolysin treatment of isolated organelles was carried out as described previously with minor modifications (Mullin et al. 2006). Intact organelles were isolated as mentioned above however the hypotonic buffer did not contain EGTA and protease inhibitors. The 4x assay buffer used was 200mM HEPES-NaOH (pH 7.4), 1.2M sorbitol $\left(\right.$ Sigma $\left.^{\circledR}\right)$ and $2 \mathrm{mM} \mathrm{CaCl}_{2}(\mathrm{Merck})$. The organellar pellet was divided into six fractions. One fraction was used for protein estimation by Bradford assay using BSA as a standard. The remaining pellets were treated as follows. i) no thermolysin, ii) $25 \mu \mathrm{g}$ thermolysin $\left(\operatorname{Sigma}^{\circledR}\right)$ per $\mathrm{mg}$ of parasite proteins, iii) $25 \mu \mathrm{g}$ thermolysin per mg of parasite proteins and 10mM EDTA (to inhibit the thermolysin), iv) $25 \mu \mathrm{g}$ thermolysin per mg of parasite proteins and 1\% Triton X-100 (to permeabilize the organelles), v) $25 \mu \mathrm{g}$ thermolysin per mg of parasite proteins, $1 \%$ Triton X-100 and 10mM EDTA. After 30 minute incubation at $30^{\circ} \mathrm{C}$, thermolysin was inhibited by adding EDTA to a final concentration of $10 \mathrm{mM}$. Protein were precipitated by chloroform/methanol/water and analyzed by Western

257 blotting.

258

259

260

261

262

$\underline{\text { Results }}$

Aluminum Tetrafluoride ( $\left.\mathrm{AlF}_{4}{ }_{4}\right)$ disrupts localization of PfEMP-1, KAHRP and PfTPx $\underline{G l}_{2}$ leaving ACP and PfUROD localization unaffected

Heterotrimeric G-proteins control the recognition and fusion between transport vesicles and their acceptor compartments (Balch 1992; Takai et al. 2001). $\mathrm{AlF}_{4}{ }^{-}$binds to the Ga subunit of G-proteins by mimicking the $\gamma$-phosphate group of GTP; as a result, the heterotrimeric G- 
264 protein remains in an active state even after GTP is hydrolysed to GDP (Chabre 1990; Finazzi et

265 al. 1994; Kahn 1991). This continuous activation inhibits ARF-mediated coatomer coat shedding 266 from vesicles. The resulting inhibition of vesicle fusion with target membranes after treatment 267 with $\mathrm{AlF}_{4}^{-}$has been demonstrated in several organisms, including Plasmodium (Taraschi et al. 268 2001). The majority of trafficking vesicles are inhibited by $\mathrm{AlF}_{4}^{-}$, the only exception so far being 269 endocytosis of CD94/NKG2A in natural killer cells (Masilamani et al. 2008).

$\mathrm{PfTPx}_{\mathrm{Gl}}$ was shown to be trafficked to the apicoplast by a Brefeldin-A sensitive pathway

271 which suggests transit through the Golgi (Chaudhari et al. 2012); these data further indicated a

272 vesicular component for targeting of $\mathrm{PfTPx}_{\mathrm{Gl}}$. For another apicoplast protein, ACP, current

273 models of trafficking have suggested that it may be transferred from the ER to the apicoplast via

274 the Golgi (Heiny et al. 2014), from the ER to the apicoplast by vesicles, or by direct transfer due

275 to transient contiguity between the membranes of the two organelles (Tonkin et al. 2006b). As

$276 \mathrm{AlF}_{4}$-inhibits vesicular fusion, these hypotheses were tested.

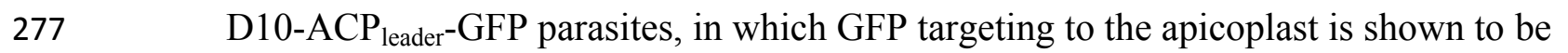

278 independent of the Golgi (Tonkin et al. 2006b), were used for treatment with $\mathrm{AlF}_{4}^{-}$and

279 localization of ACP-GFP and PfTPx $\mathrm{Gl}_{\mathrm{Gl}}$ was analyzed. Parasites were treated with $\mathrm{IC}_{50}$

280 concentration of $\mathrm{AlF}_{4}^{-}(1.2 \mu \mathrm{M}$, Fig. S1A), which did not alter parasite morphology (Fig.S1B).

281 First, the efficacy of the $\mathrm{AlF}_{4}^{-}$treatment at the $\mathrm{IC}_{50}$ concentration of $1.2 \mu \mathrm{M}$ was analyzed

282 by observing its effect on the secreted protein PfEMP1. Previously, treatment with $100 \mu \mathrm{M} \mathrm{AlF}_{4}^{-}$

283 for two hours inhibited the fusion of PfEMP1 containing vesicles with target membranes

284 (Taraschi et al. 2001). In this report, in parasites treated with $1.2 \mu \mathrm{M} \mathrm{AlF}_{4}{ }^{-}$for 18 hours, PfEMP1

285 trafficking to the host RBC was inhibited as a majority of the protein was observed in the

286 parasite (Fig. 1B, Supplementary Table S2C). This was in contrast to untreated parasites where

287 the protein was found both in the parasite and in punctate structures in the host RBC (Fig. 1A).

288 Similar results were obtained with another secretory protein KAHRP (Fig. S2, Supplementary

289 Table S2C), confirming that conditions for $\mathrm{AlF}_{4}$-treatment used in this study are robust for 290 secretory proteins.

291 Next, we studied the localization of $\mathrm{PfTPx}_{\mathrm{Gl}}$ upon treatment with $\mathrm{AlF}_{4}^{-}$. The control

292 cultures grown in parallel to the treated cultures showed apicoplast localization of

293 PfTPx $_{\mathrm{Gl}}$ between 45\% to 55\%(Supplementary Table S2A). However, in around 98\% AlF4-

294 treated parasites observed, PfTPx $_{\mathrm{Gl}}$ targeting was disrupted and punctate staining was visible 
295 throughout the parasite (Fig. 1B).No co-localization was observed with an apicoplast marker

296 protein for any of these parasites. This indicated that apicoplast localization is affected by the

297 treatment. When the parasites from the same treated cultures were analyzed for co-localization of

298 the disrupted $\mathrm{PfTPx}_{\mathrm{Gl}}$ signal with mitochondrial marker protein ferrochelatase(PfFC), we

299 observed that in $35-40 \%$ of cells the $\mathrm{PfTPx}_{\mathrm{Gl}}$ signal showed some overlap (Fig.S3). This data

300 indicated that trafficking of $\mathrm{PfTPx}_{\mathrm{Gl}}$ to the mitochondrion may be partially disrupted by the

301 treatments and requires further characterization. In contrast, as the apicoplast signal was

302 disrupted in $98 \%$ of parasites, we chose to focus on only the apicoplast localization of this

303 protein.

304 In contrast, targeting of the apicoplast marker protein ACP-GFP was not disrupted and

305 showed localization to distinct structures indicative of the organelle (Fig. 1B). To exclude the

306 possibility that these observations were an artifact of the GFP fusion of this protein, endogenous

307 ACP was also monitored in $\mathrm{AlF}_{4}^{-}$treated parasites by immunofluorescence assays. In control as

308 well as treated parasites, endogenous ACP colocalized perfectly with ACP-GFP signal

309 suggesting that it was unaltered by the treatment (Fig. 1A,B).

310 As import of ACP-GFP into the apicoplast leads to transit peptide cleavage which can be

311 monitored by Western blot (van Dooren et al. 2002), $\mathrm{AlF}_{4}$ - treated parasites (treated for $18 \pm 2$

312 hours) were subjected to this analysis. Two bands were observed on the Western blot; the

313 uppermost band represents the unprocessed form (indicated by an arrowhead) while the lower

314 band represents the processed form of ACP-GFP (Fig. 1C)(Waller et al. 2000). The majority of

315 ACP-GFP from $\mathrm{AlF}_{4}{ }^{-}$treated parasites was observed to be in the processed form, corroborating

316 the results obtained with immunofluorescence assays. As expected, PfTPx $\mathrm{Gl}_{\mathrm{Gl}}$ was not processed as

317 reported earlier (Chaudhari et al. 2012).

318 Similar experiments were done in the 3D7 parasite strain with another luminal apicoplast

319 protein Uroporphyrinogen III decarboxylase (PfUROD). Antibodies recognizing PfUROD have

320 been previously characterized (Nagaraj et al. 2009). Here, parasites treated with $1.2 \mu \mathrm{M} \mathrm{AlF}_{4}{ }^{-}$for

32118 hoursshowed PfTPx $_{\mathrm{Gl}}$ distributed throughout the cell, while the targeting of PfUROD was not

322 disrupted (Fig. S4B, Supplementary Table S2A).

323

324 Microtubule destabilizing drugs disrupt the localization of PfEMP-1, KAHRP and PfTPx $\underline{G l}_{2}$

325 leaving ACP and PfUROD localization unaffected 
Based on the potential involvement of vesicles in these trafficking of PfEMP-1, KAHRP

327

328

329

330

331

332

333

334

335

336

337

338

339

340

341

342

343

344

345

346

347

348

349

350

351

352

353

354

355

356 and PfTPx $\mathrm{Gl}_{\mathrm{Gl}}$ but not of ACP and PfUROD, microtubules were studied; these polymers play an important role in the directional trafficking of cargo via vesicles and are also vital for the positioning of organelles such as the ER and the Golgi. Microtubule destabilizing drugs collapse the ER and the Golgi, redistributing them throughout the cell(Cole \& Lippincott-Schwartz 1995). A few of these drugs like vinblsatine and nocodazole were tested previously in Plasmodium and shown to destabilize the microtubules(Chakrabarti et al. 2013). Any protein dependent on vesicular transport would be expected to be dependent on microtubule integrity.

To study the role of microtubules in targeting of proteins that use different pathways, D10-ACP $\mathrm{P}_{\text {leader }}$-GFP cells were treated with the $\mathrm{IC}_{50}$ concentration (Chakrabarti et al. 2013) of the microtubule destabilizing drug vinblastine. This concentration did not alter parasite morphology (Fig. S1B). To confirm that microtubule organization is indeed disrupted, immunofluorescence was performed with antibodies against tubulin. In control cells, intact microtubules forming hemispindles and sub-pellicular structures were observed (Fig. 2A). Upon treatment with vinblastine, the cells showed diffused staining throughout the cytosol (Fig. 2B). The disruption was reversed after the drug was washed out from the medium (Fig. 3). Similar results were observed for nocodazole (Fig. S5).

In control cells, PfEMP1 and KAHRP were efficiently exported out of the parasite and showed staining as seen in other reports(Knuepfer et al. 2005; Wickham et al. 2001). Upon treatment of cells with vinblastine, PfEMP1 and KAHRP showed an accumulation of both proteins in the parasite and decreased protein in the erythrocyte (Fig. 2, Fig.S2, Supplementary Table S2C).

When PfTPx $_{\mathrm{Gl}}$ localization was checked, the control parasites showed a staining pattern of $\operatorname{PfTPx}_{\mathrm{Gl}}$ (Fig. 2A) similar to untreated parasites where apicoplast localization was observed anywhere from $45 \%$ to $55 \%$ (Supplementary Table S2A). When the parasites were treated with vinblastine, punctate staining of $\mathrm{PfTPx}_{\mathrm{Gl}}$ was visible throughout the parasite (Fig. 2B) where apicoplast localization was disrupted in more than $94 \%$ parasites. When the drug was washed out from the medium, PfTPx $_{\mathrm{Gl}}$ was found to be co-localized with ACP-GFP within 4 hours, indicating its presence in the apicoplast (Fig. 3) in 47\% parasites(Supplementary Table S2A). Interestingly, in vinblastine-treated cells, ACP-GFP showed appropriate trafficking to the apicoplast (Fig. 2B) detected by a single spot. Detection of the endogenous ACP with antibodies 
357 showed the same results (Fig. 2A,B). Thus, the targeting of ACP is insensitive to the vinblastine 358 that disruptsPfTPx $\mathrm{X}_{\mathrm{Gl}}$ trafficking.

359 Additionally, parasites were treated with another microtubule destabilizing drug,

360

361

362

363

364

365

366

367

368

369

370

371

372

373

374

375

376

377

378

379

380

381

382

383

384

385

386

387

nocodazole (at the $\mathrm{IC}_{50}$ concentration of $17 \mu \mathrm{M}$ ). The results of ACP-GFP and PfTPx $\mathrm{Xl}_{\mathrm{Gl}}$

localization after treatment with this drug closely resembled those of vinblastine treatment (Fig.

S5), confirming that microtubules are involved in the trafficking of PfTPx $x_{\mathrm{Gl}}$ but not ACP-GFP to the apicoplast.

Similar to ACP-GFP, PfUROD trafficking to the apicoplast was not inhibited by vinblastine or nocodazole in 3D7 cells. However, in the same experiment, $\operatorname{PfTPx}_{\mathrm{Gl}}$ targeting was disrupted in the drug-treated parasites. $\mathrm{PfTPx}_{\mathrm{Gl}}$ targeting to the organelles was restored when the drugs were washed out from the medium, as observed by the co-localization of PfTPx $_{\mathrm{Gl}}$ with PfUROD(Fig. S4C,D,E,F, Supplementary Table S2A).

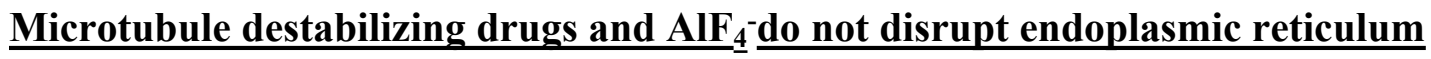
morphology but have severe effects on Golgi morphology

The role of different secretory components (G-proteins, small GTPases, cytoskeletal elements, and phosphatases) in maintaining the spatial distribution and structure of organelles has been shown in other eukaryotes with $\mathrm{AlF}_{4}{ }^{-}$and microtubule inhibitors(Back et al. 2004; Cole \& Lippincott-Schwartz 1995). These inhibitors result in ER and Golgi dispersal, finally leading to compromised secretory traffic. However, their effects on P. falciparum ER and Golgi morphology are yet not known. To understand this, and assess whether the effects are consistent with the observed disruption of $\mathrm{PfTPx}_{\mathrm{Gl}}$ localization to the apicoplast, we treated parasites with the same concentrations of $\mathrm{AlF}_{4}$-and vinblastine as described above, to study their effects on the ER and the Golgi. These organelles were visualized using antibodies against the ER-resident Binding immunoglobulin Protein (PfBiP) and Golgi reassembly and stacking protein (PfGRASP) respectively.

Unlike mammalian cells, no disintegration of the ER was observed in parasites subjected to vinblastine and $\mathrm{AlF}_{4}^{-}$. Both control parasites and drug-treated parasites showed perinuclear ER morphology consistent with the normal development of the ER (Fig. 4; control parasites A, C and treated parasites B, D, Fig. S6). As expected, in experiments where parasites were treated with $\mathrm{AlF}_{4}^{-}$or vinblastine, $\mathrm{PfTPx}_{\mathrm{Gl}}$ trafficking was severely disrupted and in some cells showed 
388 partial overlap with the ER marker PfBiP suggesting an arrest in the ER. PfTPx $x_{\mathrm{Gl}}$ targeting

389 reverted to normal when the drug was washed out from the medium (Fig. 4E).

390

In our analysis, staining of the parasites with anti-GRASP antibodies showed a pattern for

391

392

393

394

395

396

397

398

399

400

401

402

403

404

405

406

407

408

409

410

411

412

413

414

415

416

417

418

Golgi morphology that looked like a single spot surrounded by diffuse staining for some parasites (Figure 5) and for other parasites, a single spot with no diffuse staining (Supplementary Figure 7). This diffuse staining was different from previously published reports where a single spot was seen for the parasite Golgi (Struck et al. 2005; Struck et al. 2008). Thus, for Golgi staining the discrete spots are consistent with published data however, a heterogenous phenotype was observed with respect to the additional diffuse staining.

In contrast to ER staining, the same treatments resulted in dispersed Golgi staining, indicating a collapse of the Golgi morphology in more than 95\% parasites (Fig. 5B,D, Supplementary Table S2B). In control parasites, the Golgi appeared to be a distinct structure as shown in Fig. 5A,C. In drug-treated parasites, disrupted PfTPx $_{\mathrm{Gl}}$ showed partial co-localization with the disintegrated Golgi structures suggesting its arrest in the Golgi due to the drug treatments. Importantly, in drug was washed parasites Golgi morphology was reverted to normal in $90 \%$ of parasites observed (Fig. 5E).

\section{PfTPx $_{G I}$ is targeted to the outermost membrane of the apicoplast}

Proteins located on the membranes of the apicoplast do not have conventional transit peptides. Transport of these membrane-bound apicoplast proteins from the ER to the apicoplast has been proposed to occur via vesicular trafficking(Karnataki et al. 2007; Lim et al. 2009; Mullin et al. 2006). A signal anchor is thought to retain the protein in the ER membrane, following which it is targeted to the apicoplast outer membrane by vesicles (Lim et al. 2016; Mullin et al. 2006). Consistent with these observations, analysis by PlasmoAP(Foth et al. 2003) predicts that $\mathrm{PfTPx}_{\mathrm{Gl}}$ does not possess a canonical transit peptide. Interestingly, our experiments confirm that $\mathrm{PfTPx}_{\mathrm{Gl}}$ in parasites has a molecular weight suggestive of a lack of transit peptide cleavage (Chaudhari et al. 2012). These data indicate that $\mathrm{PfTP}_{\mathrm{Gl}}$ might reside on the outer membrane of the apicoplast.

The association of $\mathrm{PfTPx}_{\mathrm{Gl}}$ with organellar membranes was investigated by differential solubilization of membranes. After hypotonic lysis, most of the apicoplast luminal protein ACPGFP was extracted into the soluble fraction (Fig. 6A). The small fraction that was retained in the 
419 pellet was possibly due to incomplete lysis of the organelles. Unlike ACP-GFP, PfTPx $\mathrm{Gl}_{\mathrm{Gl}}$ was

420 found only in the pellet fraction indicating its association with the membrane (Fig. 6A).

421 To test whether $\operatorname{PfTPx}_{\mathrm{Gl}}$ is indeed localized on the outer apicoplast membrane, the

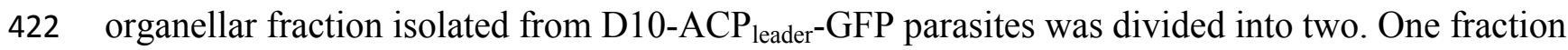

423 was not subjected to fixation and permeabilization while the second fraction was fixed and

424 permeabilized followed by immunofluorescence. ACP-GFP was used as a luminal control for

425 permeabilization since this protein should be detected by antibodies only in permeabilized

426 organelles. In contrast, antibodies would recognize $\mathrm{PfTPx}_{\mathrm{Gl}}$ in non-permeabilized organelles only

427 if it was situated on the outermost membrane.

428 As expected, anti-GFP antibodies stained the apicoplast lumen (red signal) only in the

429 permeabilized organelles, but not in the intact ones (Fig. 6C). This immunofluorescence signal

430 colocalized with the intrinsic GFP fluorescence (green signal) of ACP-GFP. In both fractions,

431 anti-PfTPx $\mathrm{Gl}_{\mathrm{Gl}}$ antibodies clearly showed staining surrounding the fluorescent signals from luminal

432 ACP-GFP (Fig. 6C). The halo around ACP-GFP, which was not observed for whole cells at

433 lower magnifications, suggested that $\mathrm{PfTPx}_{\mathrm{Gl}}$ resides on the outermost membrane of the

434 apicoplast. This observation is consistent with similar experiments conducted for other

435 membrane-bound apicoplast proteins(Kalanon et al. 2009; Mullin et al. 2006).

436 Additionally, the organellar fraction isolated from $\mathrm{AlF}_{4}$-treated parasites was probed with

437 antibodies against $\mathrm{PfTPx}_{\mathrm{Gl}}$. While the size and morphology of free intact apicoplasts (based on

438 ACP-GFP staining) remained unaltered, no peri-organellar PfTPx $\mathrm{x}_{\mathrm{Gl}}$ signal was observed in the

439 immunofluorescence analysis of intact as well as permeabilized organelles, indicating the

440 involvement of vesicles in the membrane targeting of this protein (Fig. 6D).

As $\operatorname{PfTPx}_{\mathrm{Gl}}$ is known to be dually localized to both the apicoplast and the mitochondrion,

442 it was important to confirm that the PfTPxGl staining surrounding the luminal GFP signal was

443 not mitochondrially localized protein. This was particularly important as it is known that the

444 apicoplast and mitochondrion are closely associated in P. falciparum. Staining the organellar

445 fraction with the red-fluorescent mitochondrial dye, MitoTracker showed that in our organellar

446 preparations, the MitoTracker signal was clearly distinct from the ACP-GFP signal and was not

447 seen to form a halo around the apicoplast in any microscopic field (Fig. 6B). This suggests that

448 the PfTPx $_{\mathrm{Gl}}$ staining surrounding the luminal ACP-GFP signal is indeed coming from the

449 membranes of the apicoplast and not from the mitochondrion. 
To support these findings, we treated the isolated organelles with thermolysin, a protease

451

452

453

454

455

456

457

458

459

460

461

462

463

464

465

466

467

468

469

470

471

472

473

474

475

476

477

478

479

that acts outside of intact membrane compartments. Thermolysin completely digested

$\operatorname{PfTPx}_{\mathrm{Gl}}$ showing that this protein is present on the outermost membrane. However, in these intact organelles, we found ACP-GFP largely undigested although a slightly lower amount of this protein was observed compared to controls with no thermolysin. This might be due the organellar integrity beingsomewhat compromised during handling. However, as the same experiment showed intact ACP-GFP but complete degradation of PfTPx $\mathrm{x}_{\mathrm{Gl}}$ we infer that PfTPx $\mathrm{x}_{\mathrm{Gl}}$ is localized on the outermost membrane.

As expected, $\mathrm{PfTPx}_{\mathrm{Gl}}$ was protected when the thermolysin was inhibited by the addition of EDTA. When the organelles were permeabilized by Triton X-100, both PfTPx $\mathrm{X}_{\mathrm{Gl}}$ and ACPGFP were digested (Fig. 6E). In conclusion, both immunofluorescence assays and thermolysin treatments strongly suggest that $\mathrm{PfTPx}_{\mathrm{Gl}}$ is located on the outermost membrane of the apicoplast.

\section{$\underline{\text { Discussion }}$}

\section{$\underline{P f T P x}_{\underline{G l}}$ an apicoplast membrane protein, is carried by vesicles}

In this report, $\operatorname{PfTPx}_{\mathrm{Gl}}$ is shown to be membrane-bound and appears to be on the outermost apicoplast membrane. Consistent with this data, TMPred and RHYTHM algorithms predict a trans-membrane domain in the $\mathrm{N}$-terminus of this protein. Further, an analysis of the first 60 amino acids of the N-terminal leader sequence using ProtParam tool and ExPASy revealed an enrichment of hydrophobic residues (40\%). Published data supports the membrane localization of the protein, as peptides corresponding to $\operatorname{PfTPx}_{\mathrm{Gl}}$ were found in mass spectrometric analysis of the parasite membrane proteome(Lasonder et al. 2002; Yam et al. 2013).

The membrane localization of the protein is consistent with its trafficking pathway. Transit sequences of apicoplast targeted proteins are highly enriched in positively charged residues that are recognized by organellar translocons(Foth et al. 2003; Tonkin et al. 2006a). However, the N-terminus of $\mathrm{PfTPx}_{\mathrm{Gl}}$ has an overall negative charge indicating that it would not be recognized by translocons. We have shown in this report, that $\mathrm{PfTPx}_{\mathrm{Gl}}$ is an apicoplast membrane protein whose localization is disrupted by multiple inhibitors of vesicular trafficking, 
480 suggesting that this protein is trafficked to the apicoplast membrane through vesicles. The

481 identity of these vesicles remains an area of future research.

$482 \quad$ That PfTPx $_{\mathrm{Gl}}$ employs a vesicular pathway for apicoplast localization and also transits 483 through the Golgi (Chaudhari et al. 2012)begs the question of how this protein avoids the bulk 484 flow of protein trafficking from the Golgi through the secretory route, as seen for PfEMP-1 and

485

486

487

488

489

490

491

492

493

494

495

496

497

498

499

500

501

502

503

504

505

506

507

508

509

510

KAHRP (Fig. 7). The trans-Golgi network contains an elaborate protein sorting machinery to deliver proteins to their correct destinations (Guo et al. 2014) and our data suggest that in $P$. falciparum, sorting to the apicoplast will be a key part of this machinery.

\section{Apicoplast luminal protein trafficking is independent of vesicles}

Trafficking of ACP-GFP in P. falciparum has been shown to proceed through the ER to the apicoplast(Tonkin et al. 2006b)and this model has led to speculations about vesicles or direct contacts between organelles(Kalanon \& McFadden 2010; Lim et al. 2009; Parsons et al. 2009; Tonkin et al. 2008). Data presented here indicates that trafficking of three luminal apicoplast proteins (ACP-GFP, endogenous ACP and PfUROD) is not inhibited by blocking the fusion of G-protein dependent vesicles. Additionally, trafficking of these proteins is insensitive to microtubule destabilizing drugs such as vinblastine and nocodazole, indicating that the microtubule tracks used for classical vesicular trafficking are not essential for their transport.

A trivial explanation for these results could be that ACP-GFP, ACP and UROD have been trafficked to the apicoplast early during the asexual cycle of the parasite (during ring stages) and are highly stable proteins with limited turnover. This would result in protein localization that is insensitive to disruption with small molecules as there is no trafficking during the time of treatment. Evidence against this possibility exists in the literature. Pulse chase experiments for ACP-GFP carried out in the late ring/early trophozoite stages show that newly synthesized, unprocessed protein can be seen at these stages(Heiny et al. 2014; Tonkin et al. 2006b; van Dooren et al. 2002; Waller et al. 2000). Therefore, our treatments of 18 hours, encompassing the rings and early trophozoites, overlap with the synthesis and trafficking window for ACP-GFP. Similarly, Western blots of ACP-GFP after 18 hours of $\mathrm{AlF}_{4}^{-}$treatment also show a fraction of unprocessed protein, similar to untreated parasites (Fig. 1C).

Based on our data, we speculate that luminal apicoplast proteins are directly trafficked from the ER to the apicoplast without G-protein coupled vesicles. That ACP-GFP may be 
511 trafficked via vesicles that are insensitive to $\mathrm{AlF}_{4}{ }^{-c a n n o t}$ be excluded; although highly unusual,

512 such vesicles have been reported for the endocytosis of CD94/NKG2A in natural killer cells

513 (Masilamani et al. 2008). Interestingly, a recent model shows an ER-Golgi route for ACP-GFP

514 (Heiny et al. 2014)which implicates vesicles in trafficking of apicoplast proteins. Data from this

515 report suggest that, for this model too, G-protein dependent vesicles may not play a major role.

516 The use of microtubule destabilizing drugs has also added more insights into the unusual

517 structure of the parasite ER and Golgi. Apart from acting as the tracks for secretory traffic,

518 microtubules are also involved in the positioning and structural integrity of the ER and the Golgi

519 in mammalian cells (Cole \& Lippincott-Schwartz 1995). However, the ER and the Golgi in $P$.

520 falciparum appear remarkably different from those observed in mammalian cells (Struck et al.

521 2005; van Dooren et al. 2005). Here, we show that disruption of microtubules in P. falciparum

522 destabilizes the Golgi, while, unlike in mammalian cells, the ER does not show gross

523 morphological changes. However, the arrest of PfTPx $\mathrm{X}_{\mathrm{Gl}}$ in the ER and Golgi in treated cells

524 shows that the ER function of protein trafficking through vesicles might be compromised.

525 Interestingly, ACP-GFP localization to the apicoplast is unaffected in these experiments.

526 Proteins with the same destination (apicoplast) are trafficked by different routes (Fig. 7).

527 While PfTPx $\mathrm{Gl}_{\mathrm{Gl}}$ appears to be carried to the Golgi by the bulk flow of secretory traffic, ACP and

528 UROD are diverted from this pathway, possibly by ER receptors that recognize the transit

529 peptide(Tonkin et al. 2006b)which is lacking in $\mathrm{PfTPx}_{\mathrm{Gl}}$. Therefore, the sorting station for

$530 \operatorname{PfTPx}_{\mathrm{Gl}}$ appears to be the Golgi; for ACP and UROD it appears to be the ER. It is noteworthy

531 that differential targeting pathways for the luminal and membrane proteins of the apicoplast have

532 been shown in related Apicomplexan parasite Toxoplasma gondii as well (Bouchut et al. 2014).

533

534 Conclusions

535 In this study, we show that in P. falciparum, two different pathways exist for the

536 localization of proteins to the apicoplast. These findings raise interesting questions regarding the

537 molecular nature of the choices made by the parasite to direct proteins via one pathway or

538 another (Fig. 7). Our data suggests that one of the signals may include the absence or presence of

539 membrane anchors. A detailed understanding of these signals on proteins as well as receptors in

540 the ER, Golgi and vesicles remain areas for future studies.

541 Acknowledgements 
542 We thank Samir Jadhav, SachinTawade, Sudesh Kumar Roy at IIT Bombay, Krishanu Ray at

543 TIFR Mumbai for the help with confocal microscopy, Angus Bell at Trinity College Dublin for 544 providing the marker antibodies against PfTubulin, Neel Sarovar Bhavesh at ICGEB Delhi for 545 providing the antibodies against PfEMP1 and PfKAHRP and Chetan Chitnis at and Pawan

546 Malhotra at ICGEB for providing antibodies against PfBiP and PfGRASP.

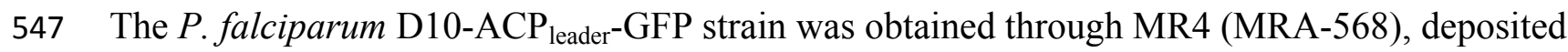
548 by G. I. McFadden.

549

550

551

552

553

554

555

556

557

558

559

560

561

562

563

564

565

566

567

568

569

570

571

\section{$\underline{\text { References }}$}

Back N, Litonius E, Mains RE, and Eipper BA. 2004. Fluoride causes reversible dispersal of Golgi cisternae and matrix in neuroendocrine cells. Eur J Cell Biol 83:389-402. 10.1078/0171-9335-00405

Balch WE. 1992. From G minor to G major. Curr Biol 2:157-160.

Bouchut A, Geiger JA, DeRocher AE, and Parsons M. 2014. Vesicles bearing Toxoplasma apicoplast membrane proteins persist following loss of the relict plastid or Golgi body disruption. PLoS One 9:e112096. 10.1371/journal.pone.0112096

Chabre M. 1990. Aluminofluoride and beryllofluoride complexes: a new phosphate analogs in enzymology. Trends Biochem Sci 15:6-10.

Chakrabarti R, Rawat PS, Cooke BM, Coppel RL, and Patankar S. 2013. Cellular effects of curcumin on Plasmodium falciparum include disruption of microtubules. PLoS One 8:e57302. 10.1371/journal.pone.0057302

Chaudhari R, Narayan A, and Patankar S. 2012. A novel trafficking pathway in Plasmodium falciparum for the organellar localization of glutathione peroxidase-like thioredoxin peroxidase. FEBS J. 10.1111/j.1742-4658.2012.08746.x

Cole NB, and Lippincott-Schwartz J. 1995. Organization of organelles and membrane traffic by microtubules. Curr Opin Cell Biol 7:55-64.

Corish P, and Tyler-Smith C. 1999. Attenuation of green fluorescent protein half-life in mammalian cells. Protein Eng 12:1035-1040. 
572 Deponte M, Hoppe HC, Lee M, Maier AG, Richard D, Rug M, Spielmann T, and Przyborski JM. 573 2012. Wherever I may roam: Protein and membrane trafficking in P. falciparum-infected 574 red blood cells. Mol Biochem Parasitol. 10.1016/j.molbiopara.2012.09.007

575

Finazzi D, Cassel D, Donaldson JG, and Klausner RD. 1994. Aluminum fluoride acts on the 576 reversibility of ARF1-dependent coat protein binding to Golgi membranes. J Biol Chem

577 269:13325-13330.

578

579

Halter M, Tona A, Bhadriraju K, Plant AL, and Elliott JT. 2007. Automated live cell imaging of green fluorescent protein degradation in individual fibroblasts. Cytometry A 71:827-834.

Foth BJ, Ralph SA, Tonkin CJ, Struck NS, Fraunholz M, Roos DS, Cowman AF, and McFadden GI. 2003. Dissecting apicoplast targeting in the malaria parasite Plasmodium falciparum. Science 299:705-708. 10.1126/science.1078599

Guo Y, Sirkis DW, and Schekman R. 2014. Protein sorting at the trans-Golgi network. Annu Rev Cell Dev Biol 30:169-206. 10.1146/annurev-cellbio-100913-013012 10.1002/cyto.a.20461

Heiny SR, Pautz S, Recker M, and Przyborski JM. 2014. Protein Traffic to the Plasmodium 587 falciparum apicoplast: evidence for a sorting branch point at the Golgi. Traffic 15:12901304. $10.1111 /$ tra. 12226

Kaderi Kibria KM, Rawat K, Klinger CM, Datta G, Panchal M, Singh S, Iyer GR, Kaur I, 590

Kahn RA. 1991. Fluoride is not an activator of the smaller (20-25 kDa) GTP-binding proteins. $J$ Biol Chem 266:15595-15597.

Kalanon M, and McFadden GI. 2010. Malaria, Plasmodium falciparum and its apicoplast. Biochem Soc Trans 38:775-782. 10.1042/BST0380775 complex 1 in protein targeting to rhoptry organelles in Plasmodium falciparum. Biochim Biophys Acta 1854:699-710. 10.1016/ju.bbamcr.2014.12.030

600 601 602
Kalanon M, Tonkin CJ, and McFadden GI. 2009. Characterization of two putative protein translocation components in the apicoplast of Plasmodium falciparum. Eukaryot Cell 8:1146-1154. 10.1128/EC.00061-09

Karnataki A, Derocher A, Coppens I, Nash C, Feagin JE, and Parsons M. 2007. Cell cycleregulated vesicular trafficking of Toxoplasma APT1, a protein localized to multiple apicoplast membranes. Mol Microbiol 63:1653-1668. 10.1111/j.1365-2958.2007.05619.x 
603 Kehr S, Sturm N, Rahlfs S, Przyborski JM, and Becker K. 2010. Compartmentation of redox 604 metabolism in malaria parasites. PLoS Pathog 6:e1001242.

$605 \quad$ 10.1371/journal.ppat.1001242

606 Knuepfer E, Rug M, Klonis N, Tilley L, and Cowman AF. 2005. Trafficking of the major 607 virulence factor to the surface of transfected P. falciparum-infected erythrocytes. Blood $608 \quad 105: 4078-4087.10 .1182 /$ blood-2004-12-4666

609 Lasonder E, Ishihama Y, Andersen JS, Vermunt AM, Pain A, Sauerwein RW, Eling WM, Hall 610 N, Waters AP, Stunnenberg HG, and Mann M. 2002. Analysis of the Plasmodium 611 falciparum proteome by high-accuracy mass spectrometry. Nature 419:537-542. $612 \quad 10.1038 /$ nature01111

613 Lemgruber L, Kudryashev M, Dekiwadia C, Riglar DT, Baum J, Stahlberg H, Ralph SA, and 614 Frischknecht F. 2013. Cryo-electron tomography reveals four-membrane architecture of 615 the Plasmodium apicoplast. Malar J 12:25. 10.1186/1475-2875-12-25

616 Lim L, Kalanon M, and McFadden GI. 2009. New proteins in the apicoplast membranes: time to 617 rethink apicoplast protein targeting. Trends Parasitol 25:197-200.

$618 \quad$ 10.1016/j.pt.2009.02.001

619 Lim L, Sayers CP, Goodman CD, and McFadden GI. 2016. Targeting of a Transporter to the 620 Outer Apicoplast Membrane in the Human Malaria Parasite Plasmodium falciparum. $621 \quad$ PLoS One 11:e0159603. 10.1371/journal.pone.0159603

622 Marechal E, and Cesbron-Delauw MF. 2001. The apicoplast: a new member of the plastid 623 family. Trends Plant Sci 6:200-205.

624 Masilamani M, Narayanan S, Prieto M, Borrego F, and Coligan JE. 2008. Uncommon endocytic 625 and trafficking pathway of the natural killer cell CD94/NKG2A inhibitory receptor.

626 Traffic 9:1019-1034. 10.1111/j.1600-0854.2008.00738.x

627 McFadden GI, and Roos DS. 1999. Apicomplexan plastids as drug targets. Trends Microbiol $628 \quad 7: 328-333$.

629 Moxon CA, Grau GE, and Craig AG. 2011. Malaria: modification of the red blood cell and 630 consequences in the human host. Br J Haematol. 10.1111/j.1365-2141.2011.08755.x 631 Mullin KA, Lim L, Ralph SA, Spurck TP, Handman E, and McFadden GI. 2006. Membrane 632 transporters in the relict plastid of malaria parasites. Proc Natl Acad Sci U S A 103:9572633 9577. 10.1073/pnas.0602293103 
634 Nagaraj VA, Arumugam R, Chandra NR, Prasad D, Rangarajan PN, and Padmanaban G. 2009. 635 Localisation of Plasmodium falciparum uroporphyrinogen III decarboxylase of the heme636 biosynthetic pathway in the apicoplast and characterisation of its catalytic properties. Int 637 J Parasitol 39:559-568. 10.1016/jd.ijpara.2008.10.011

Parsons M, Karnataki A, and Derocher AE. 2009. Evolving insights into protein trafficking to the multiple compartments of the apicomplexan plastid. J Eukaryot Microbiol 56:214220. $10.1111 /$ j.1550-7408.2009.00405.x

Struck NS, de Souza Dias S, Langer C, Marti M, Pearce JA, Cowman AF, and Gilberger TW. 642 2005. Re-defining the Golgi complex in Plasmodium falciparum using the novel Golgi marker PfGRASP. J Cell Sci 118:5603-5613. 10.1242/jcs.02673

Struck NS, Herrmann S, Schmuck-Barkmann I, de Souza Dias S, Haase S, Cabrera AL, Treeck M, Bruns C, Langer C, Cowman AF, Marti M, Spielmann T, and Gilberger TW. 2008. Spatial dissection of the cis- and trans-Golgi compartments in the malaria parasite

Takai Y, Sasaki T, and Matozaki T. 2001. Small GTP-binding proteins. Physiol Rev 81:153-208. trafficking of parasite proteins to the host cell cytosol and erythrocyte surface membrane in Plasmodium falciparum infected erythrocytes. Int J Parasitol 31:1381-1391.

Tonkin CJ, Kalanon M, and McFadden GI. 2008. Protein targeting to the malaria parasite plastid. 654 Traffic 9:166-175. 10.1111/j.1600-0854.2007.00660.x

Tonkin CJ, Roos DS, and McFadden GI. 2006a. N-terminal positively charged amino acids, but 656 not their exact position, are important for apicoplast transit peptide fidelity in Toxoplasma gondii. Mol Biochem Parasitol 150:192-200.

Tonkin CJ, Struck NS, Mullin KA, Stimmler LM, and McFadden GI. 2006b. Evidence for Golgiindependent transport from the early secretory pathway to the plastid in malaria parasites.

662 Tonkin CJ, van Dooren GG, Spurck TP, Struck NS, Good RT, Handman E, Cowman AF, and 663 McFadden GI. 2004. Localization of organellar proteins in Plasmodium falciparum using 
664

665

666

667

668

669

670

671

672

673

674

675

676

677

678

679

680

681

682

683

684

685

686 a novel set of transfection vectors and a new immunofluorescence fixation method. Mol Biochem Parasitol 137:13-21. 10.1016/j.molbiozpara.2004.05.009

van Dooren GG, Marti M, Tonkin CJ, Stimmler LM, Cowman AF, and McFadden GI. 2005. Development of the endoplasmic reticulum, mitochondrion and apicoplast during the asexual life cycle of Plasmodium falciparum. Mol Microbiol 57:405-419. 10.1111/j.1365-2958.2005.04699.x

van Dooren GG, Su V, D'Ombrain MC, and McFadden GI. 2002. Processing of an apicoplast leader sequence in Plasmodium falciparum and the identification of a putative leader cleavage enzyme. J Biol Chem 277:23612-23619. 10.1074/jbc.M201748200

Waller RF, Reed MB, Cowman AF, and McFadden GI. 2000. Protein trafficking to the plastid of Plasmodium falciparum is via the secretory pathway. EMBO J 19:1794-1802. 10.1093/emboj/19.8.1794

Wickham ME, Rug M, Ralph SA, Klonis N, McFadden GI, Tilley L, and Cowman AF. 2001. Trafficking and assembly of the cytoadherence complex in Plasmodium falciparuminfected human erythrocytes. EMBO J 20:5636-5649. 10.1093/emboj/20.20.5636

Yam XY, Birago C, Fratini F, Di Girolamo F, Raggi C, Sargiacomo M, Bachi A, Berry L, Fall G, Curra C, Pizzi E, Breton CB, and Ponzi M. 2013. Proteomic analysis of detergentresistant membrane microdomains in trophozoite blood stage of the human malaria parasite Plasmodium falciparum. Mol Cell Proteomics 12:3948-3961. 10.1074/mcp.M113.029272 


\section{Figure 1}

Immunofluorescence images show PfTPx ${ }_{G 1}$, microtubules, ACP-GFP, PfACP and PfEMP1 trafficking in $\mathrm{AlF}_{4}$-treated D10-ACPleader-GFP parasites.

(A) PfTPx ${ }_{G l}$, ACP-GFP, PfACP and PfEMP1 localization in control parasites, (B) PfTPx ${ }_{G}$, ACP-GFP, PfACP and PfEMP1 localization in $\mathrm{AlF}_{4}$-treated parasites. For D10-ACPleader-GFP parasites, 98\% of the 115 parasites analyzed showed disrupted $\mathrm{PfTPx}_{\mathrm{Gl}}$ signal while $96 \%$ of the 147 parasites analyzed showed arrest of PfEMP1 in the parasites. Scale Bar: $10 \mu \mathrm{m},(\mathrm{C})$ Processing of apicoplast targeted protein ACP-GFP is not affected in drug-treated parasites. Western blot showing the processing of apicoplast targeted ACP-GFP and PfTPx ${ }_{G I}$ in AlF $_{4}^{-}$treated parasites. In ACP-GFP panel, upper band indicated with an arrowhead represents an unprocessed form of ACP-GFP while lower band represents processed form of ACP-GFP.
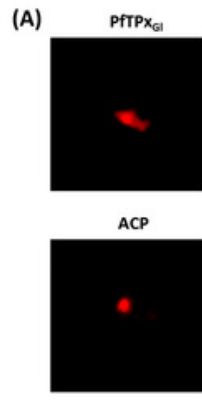

PfEMP1

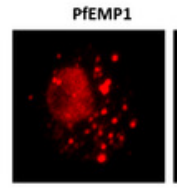

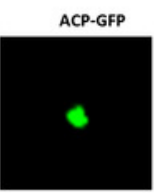

ACP-GFP

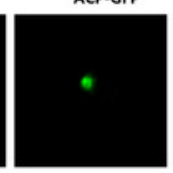

ACP-GFP

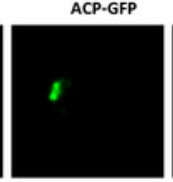

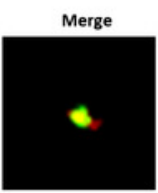

Merge

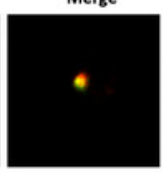

Merge

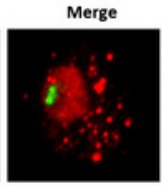

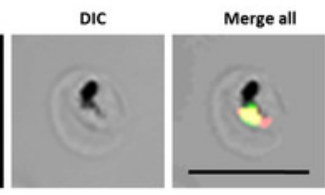

DIC

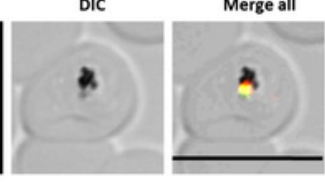

DIC

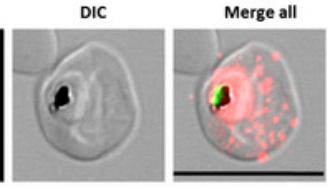

(B)
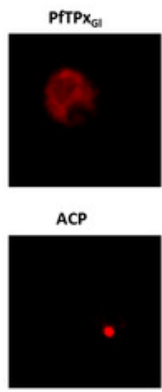

PfEMP1

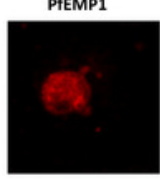

ACP-GFP

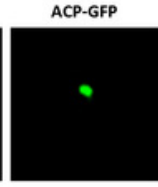

ACP-GFP
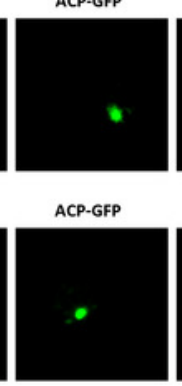

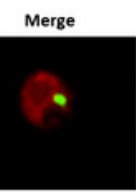

Merge

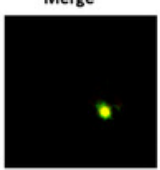

Merge

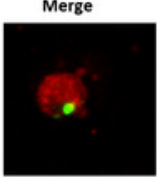

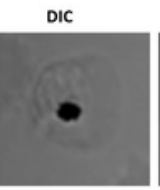

Merge all

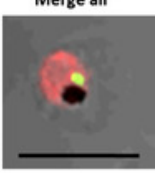

Merge all

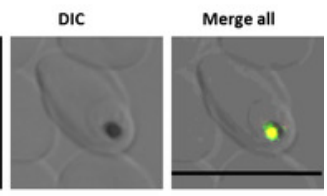

DIC

Merge all

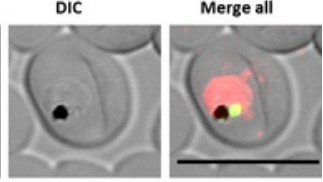

(C)

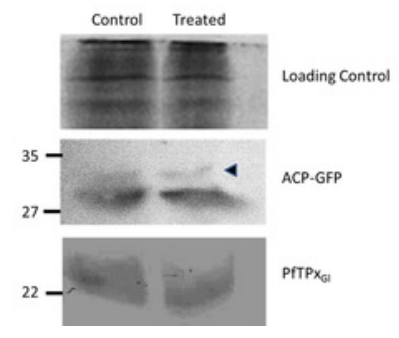




\section{Figure 2}

Immunofluorescence images show PfTP ${ }_{G}$, microtubules, ACP-GFP, PfACP and PfEMP1

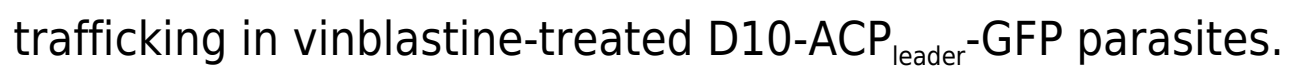

(A) PfTPx ${ }_{G l}$, microtubules, ACP-GFP, PfACP and PfEMP1 localization in solvent control parasites, (B) PfTPx ${ }_{G 1}$, microtubules, ACP-GFP, PfACP and PfEMP1 localization in vinblastine treated parasites. In these experiments, targeting to the apicoplast was inhibited in $94 \%$ of the parasites with vinblastine treatment (33 parasites counted) while an arrest of PfEMP1 was observed in $97 \%$ of the parasites (134 parasites counted). Scale Bar: $10 \mu \mathrm{m}$. 
(A)
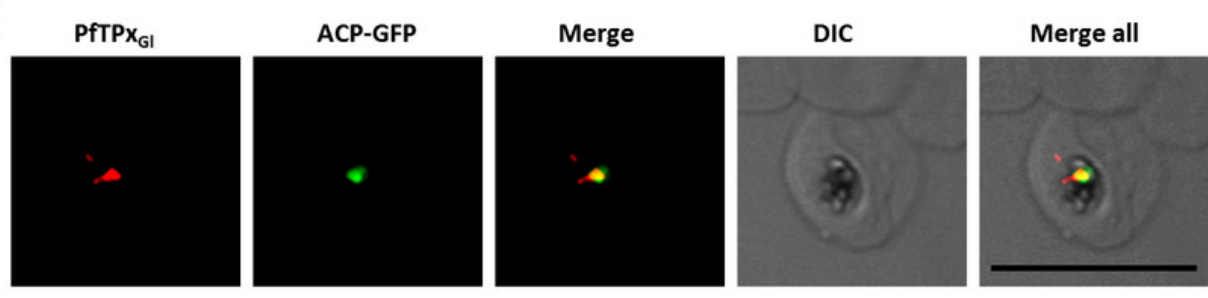

$\alpha$ - Tubulin

ACP-GFP

Merge

DIC
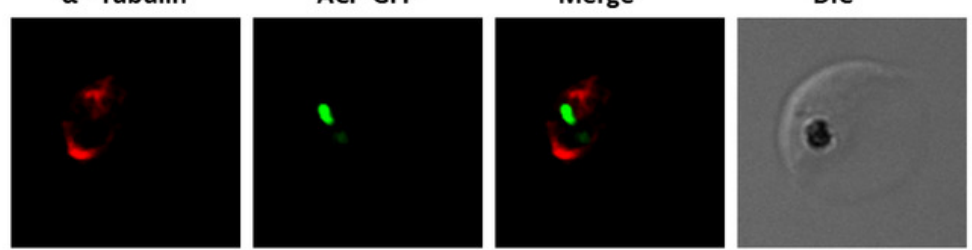

Merge all

ACP

ACP-GFP

Merge
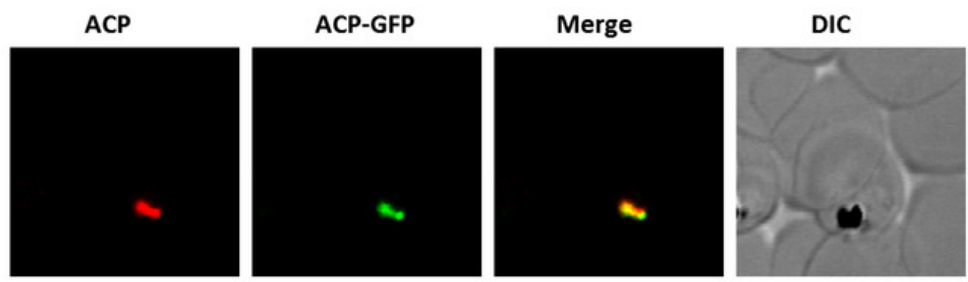

Merge all

PfEMP1

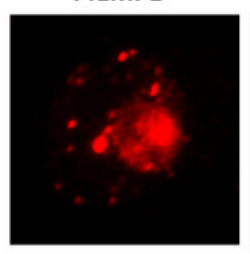

ACP-GFP

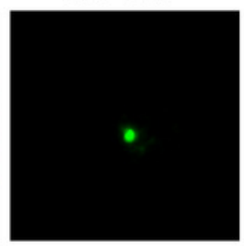

Merge
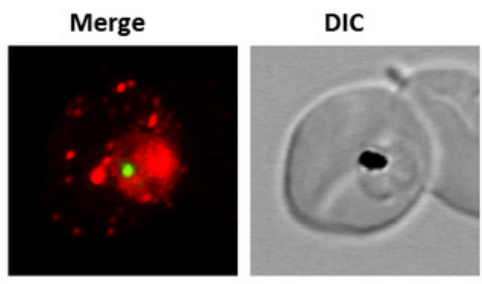

Merge all

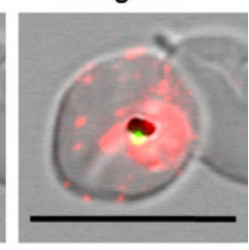

(B)
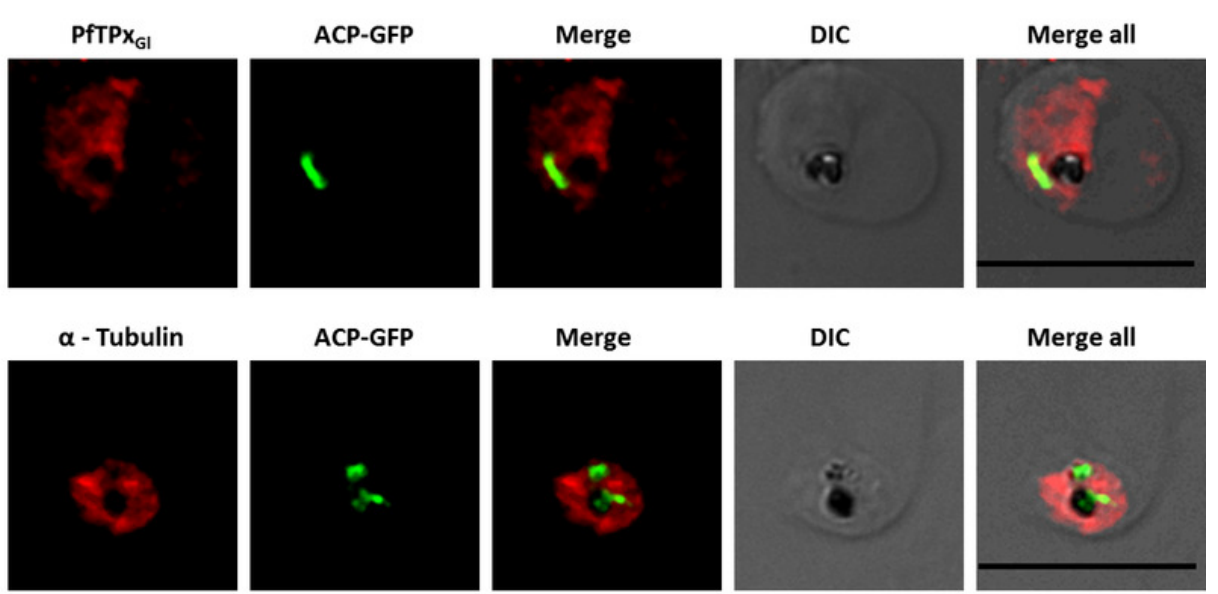

Merge all

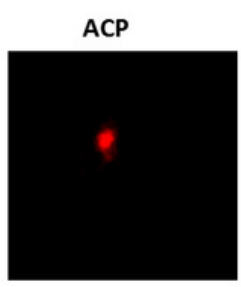

ACP-GFP

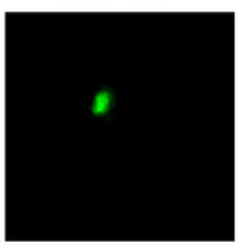

Merge
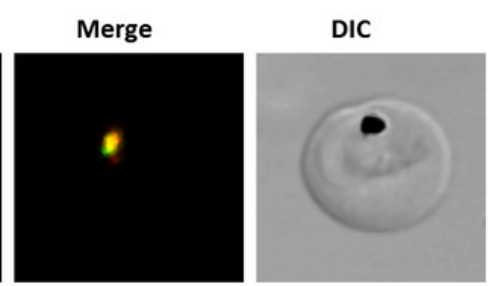

PfEMP1

\begin{abstract}
ACP-GFP
\end{abstract}

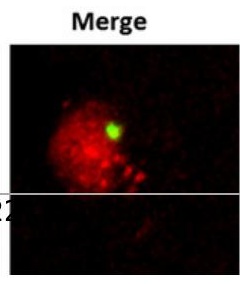

Merge all
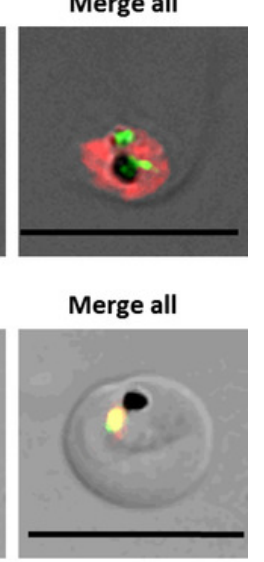

Merge all

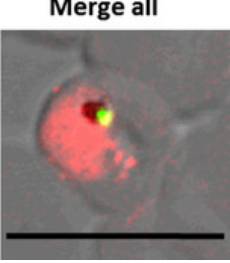




\section{Figure 3}

Immunofluorescence images showing PfTPx ${ }_{G l}$ and microtubules in D10-ACPleader-GFP parasites with drug washed out.

Reversion of $\mathrm{PfTPx}_{\mathrm{GI}}$ localization to the organelles and intact microtubular structures observed in parasites in drug washed out medium after vinblastine treatment. In these experiments, localization of $\mathrm{PfTPx}_{\mathrm{GI}}$ was reverted to the apicoplast in $47 \%$ parasites, while remaining $53 \%$ parasites showed mitochondrial localization (23 parasites counted). Scale Bar: $10 \mu \mathrm{m}$.
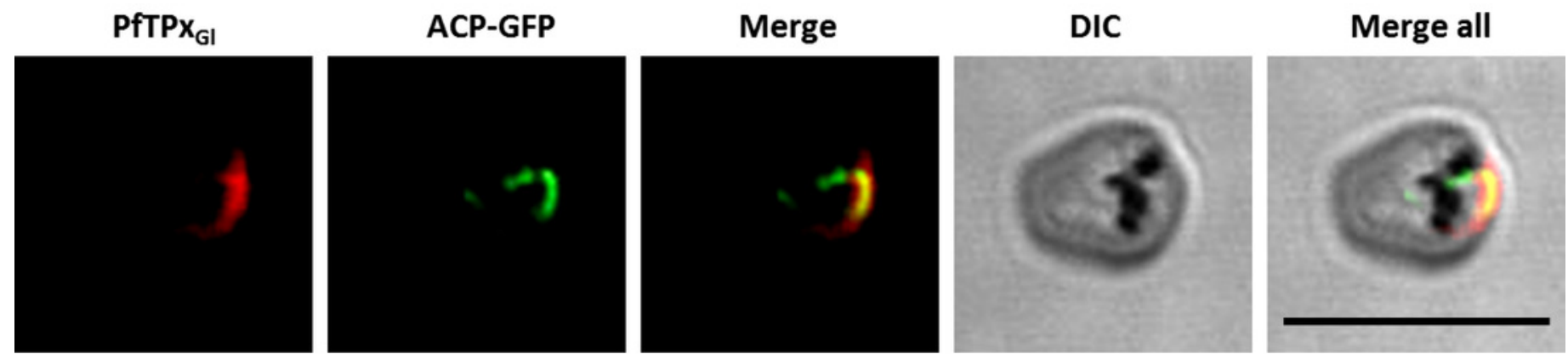

$\alpha-$ Tubulin

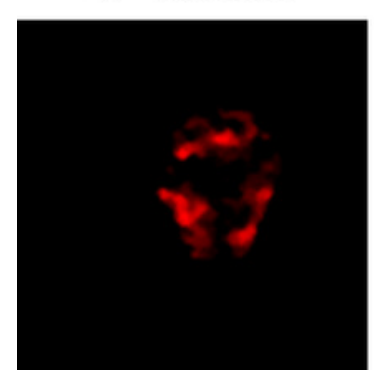

ACP-GFP

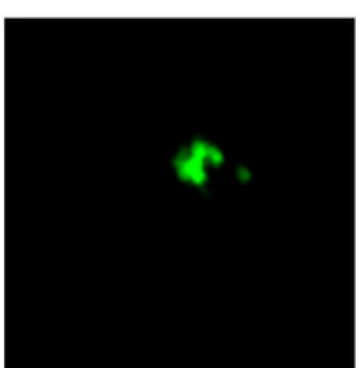

Merge

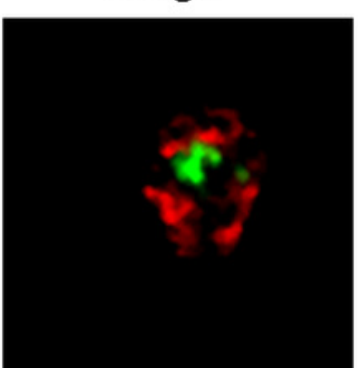

DIC

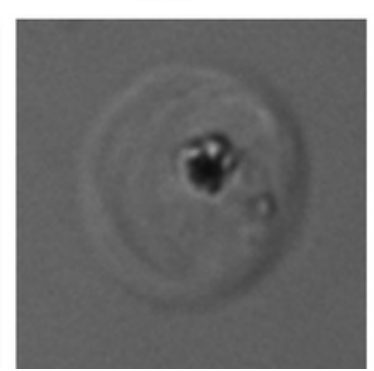

Merge all

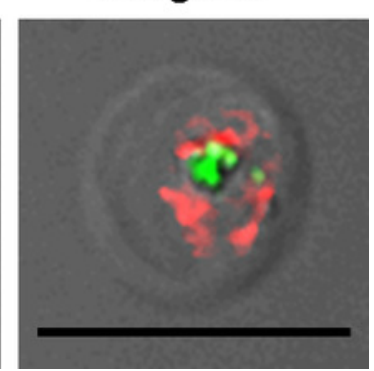




\section{Figure 4}

Immunofluorescence images showing the endoplasmic reticulum (ER) morphology in $\mathrm{AlF}_{4}$ and vinblastine treated parasites.

(A) PfBiP localization in control parasites for $\mathrm{AlF}_{4}{ }_{4}$ treatment, (B) ER morphology in $\mathrm{AlF}_{4}$ treated parasites (14 parasites were counted, none showed dispersal of ER structure), (C) PfBiP localization in solvent (PBS) control parasites for vinblastine treatment, (D) ER morphology in vinblastine-treated parasites (26 parasites were counted, none showed dispersal), (E) ER morphology in parasites reverted after vinblastine treatment (27 parasites counted). Scale Bar: $10 \mu \mathrm{m}$. 
(A)

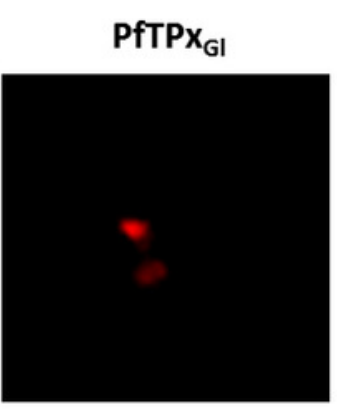

(B)

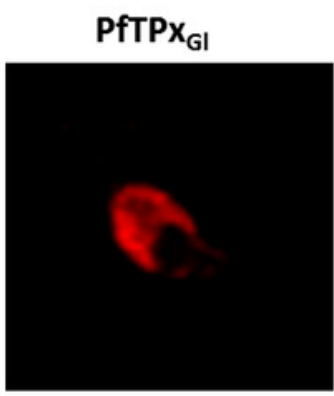

(C)

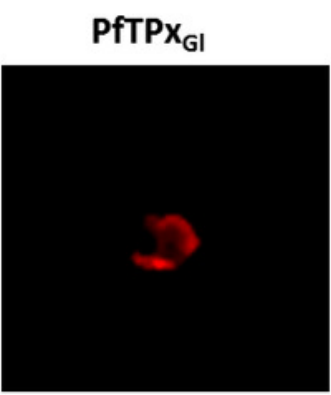

(D)

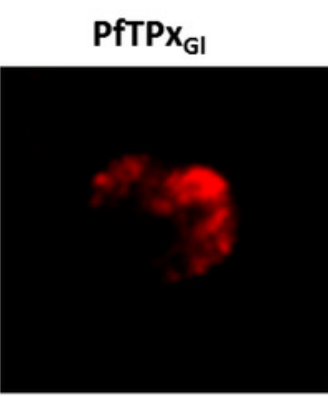

(E)

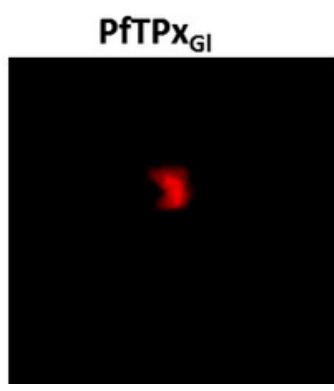

PfBip

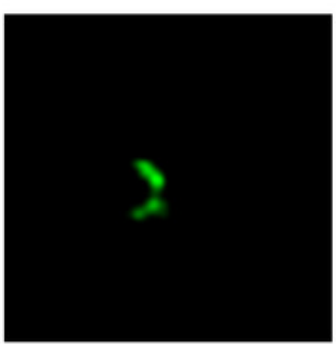

PfBip

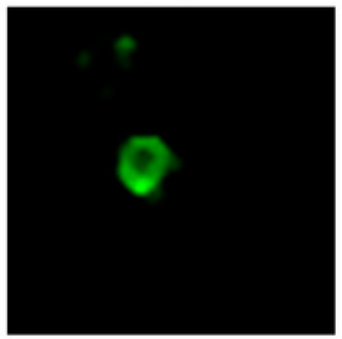

PfBip

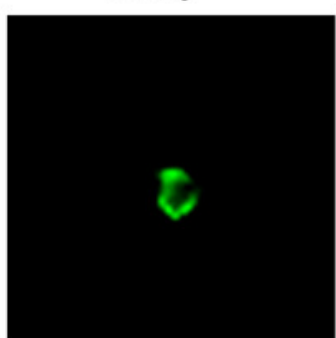

PfBip

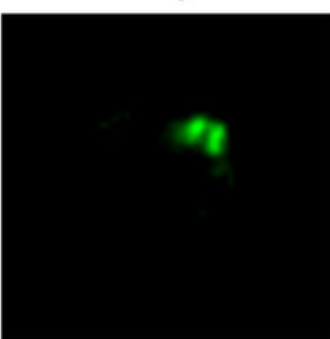

PfBip

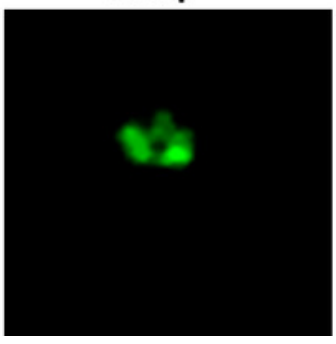

Merge

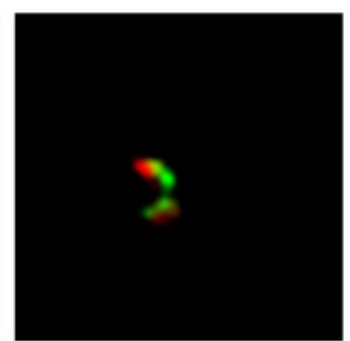

Merge

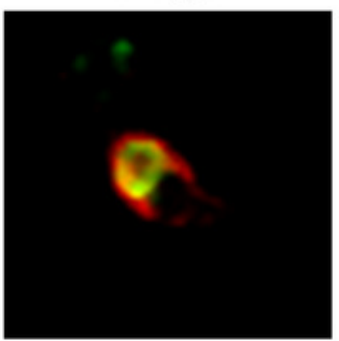

Merge

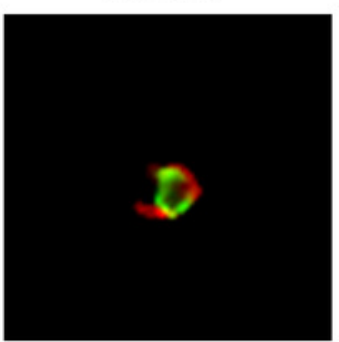

Merge

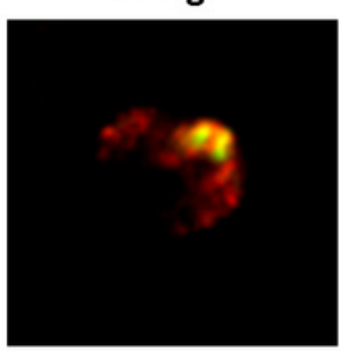

Merge

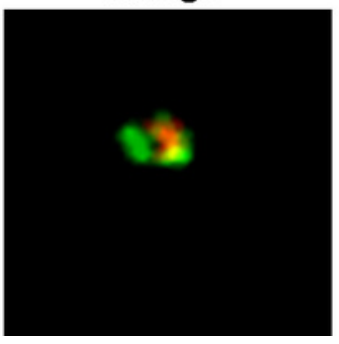

DIC

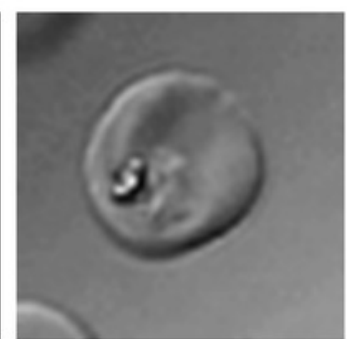

DIC

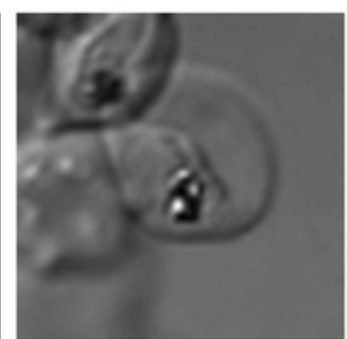

DIC

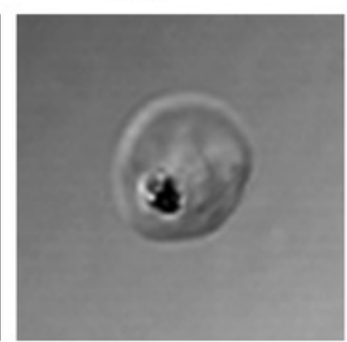

DIC

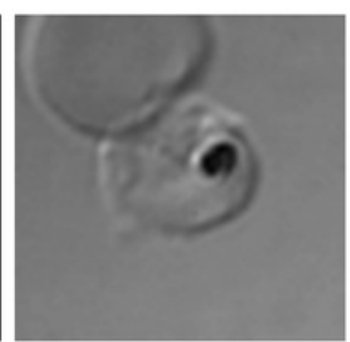

DIC

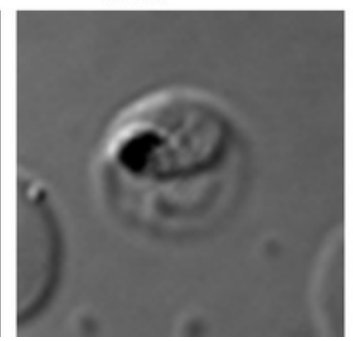

Merge all

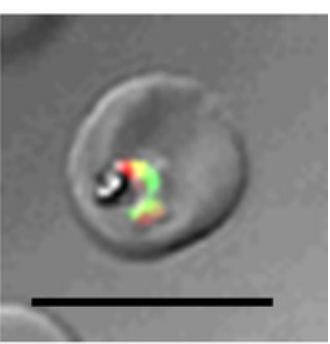

Merge all

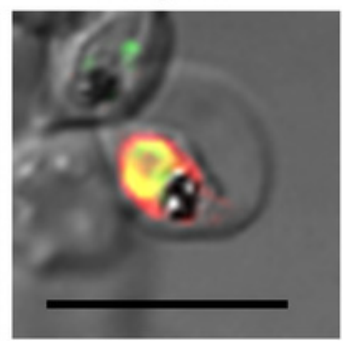

Merge all

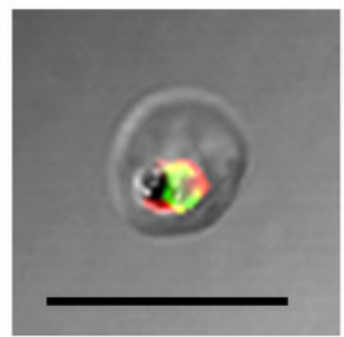

Merge all

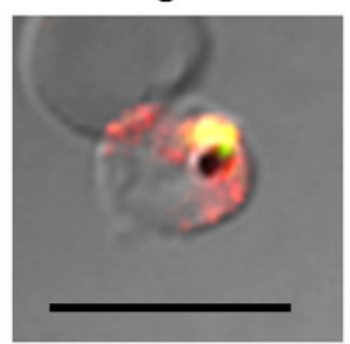

Merge all

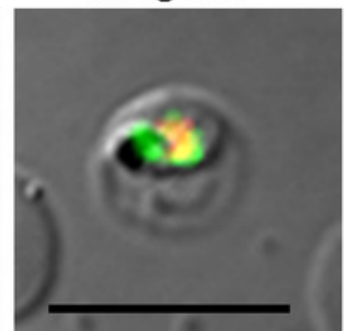




\section{Figure 5}

Immunofluorescence images showing the Golgi morphology in $\mathrm{AlF}_{4}{ }^{-}$and vinblastine treated parasites.

(A) PfGRASP localization in control parasites for $\mathrm{AlF}_{4}{ }^{-}$treatment, (B) Golgi morphology in $\mathrm{AlF}_{4}$ treated parasites (17 parasites counted, Golgi structure was dispersed in 95\% parasites), (C) PfGRASP localization in solvent (PBS) control parasites for vinblastine treatment, (D) Golgi morphology in vinblastine-treated parasites (18 parasites counted, Golgi structure was dispersed in 95\% parasites), (E) Golgi morphology in parasites reverted after vinblastine treatment (11 parasites counted, Intact Golgi structure was observed in 90\% parasites). Scale Bar: $10 \mu \mathrm{m}$. 
(A)

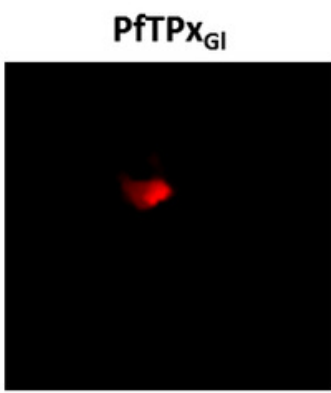

(B)

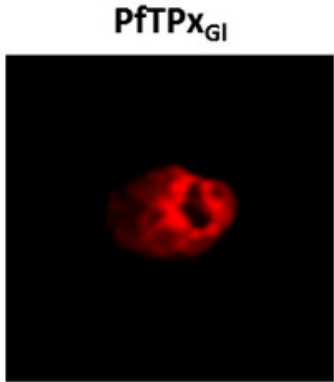

(C)

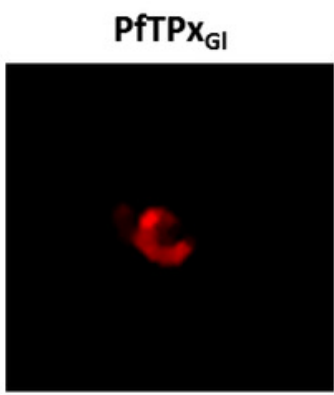

(D)

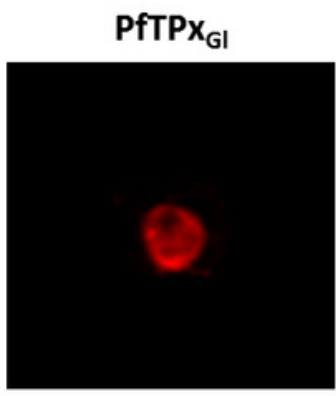

(E)

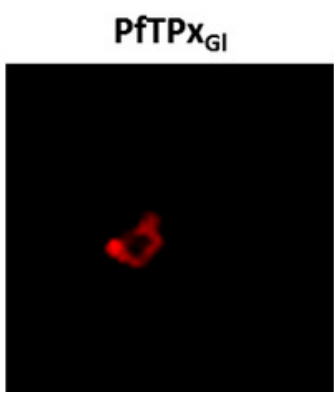

PfGRASP

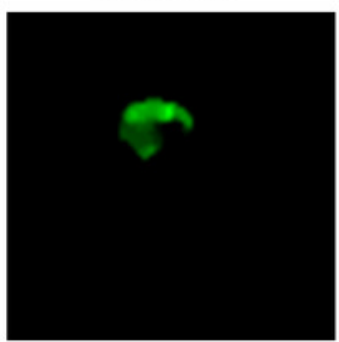

PfGRASP

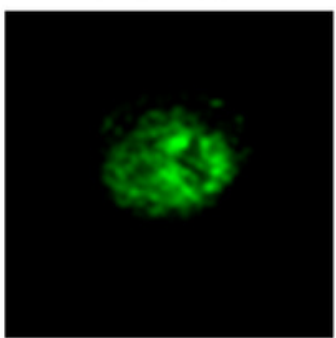

PfGRASP

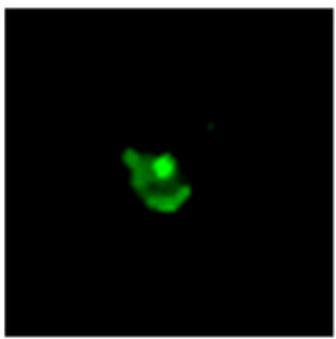

PfGRASP

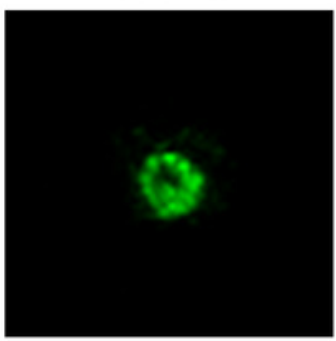

PfGRASP

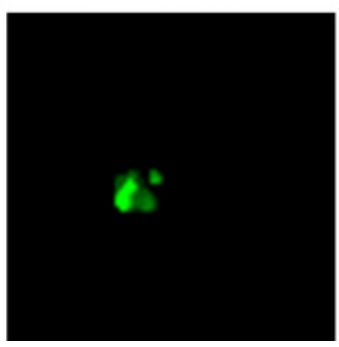

Merge

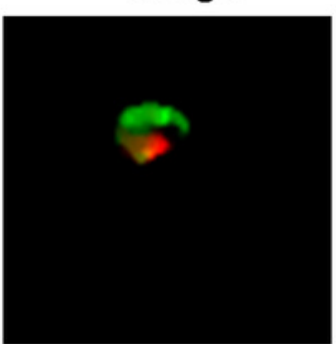

Merge

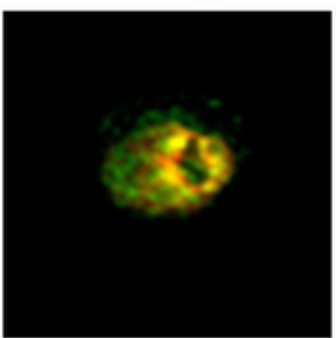

Merge

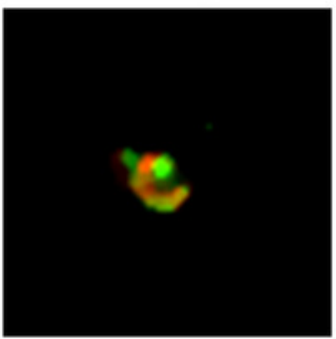

Merge

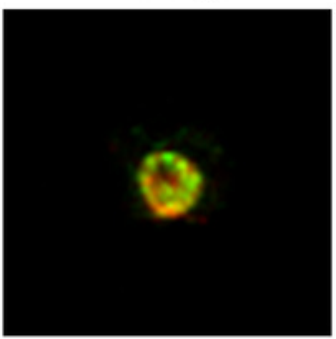

Merge

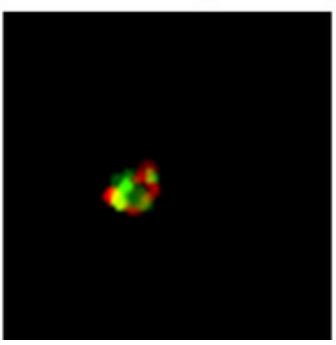

DIC

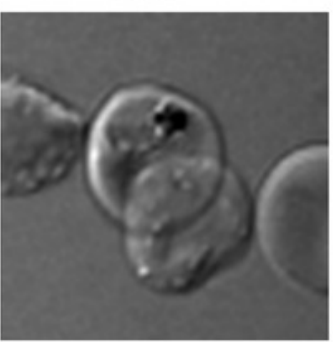

DIC

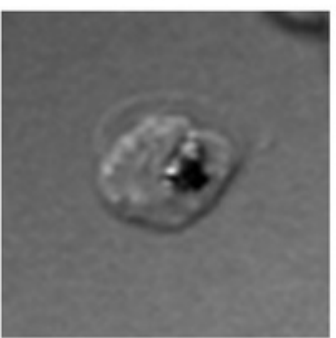

DIC

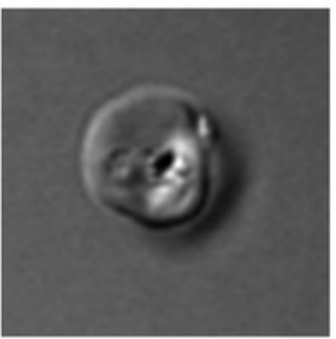

DIC

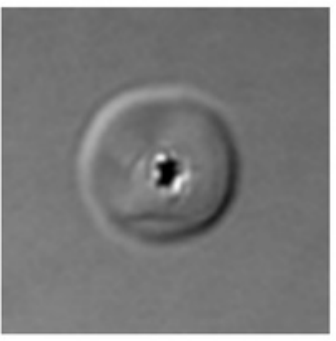

DIC

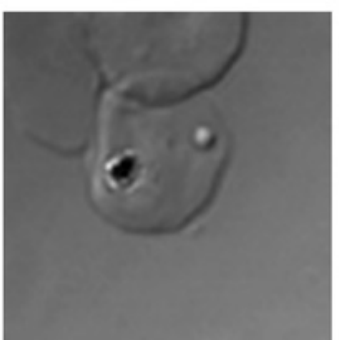

Merge all

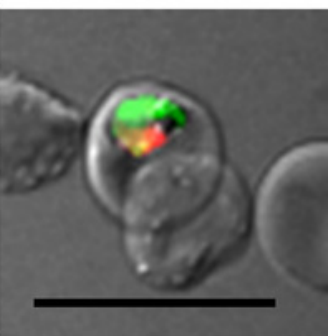

Merge all

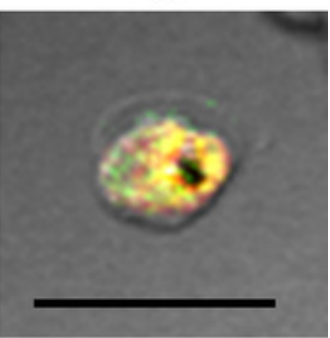

Merge all

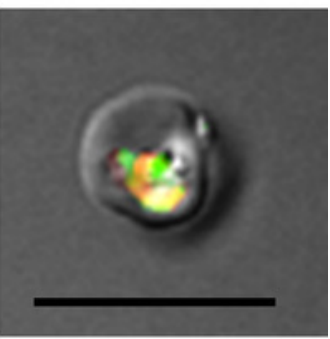

Merge all

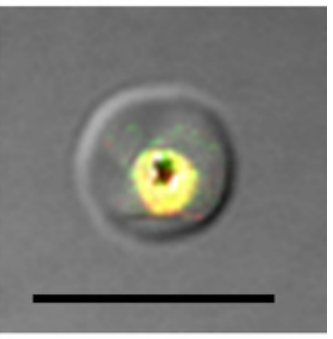

Merge all

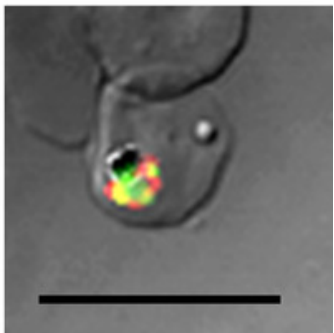




\section{Figure 6}

PfTPx $_{G I}$ localization to the outermost membrane of the apicoplast in D10-ACPleader-GFP parasites.

(A) Western blots showing association of $\mathrm{PfTP}_{\mathrm{GI}}$ with the organellar membranes (S Supernatant, $\mathrm{P}$ - Pellet) after hypotonic lysis, (B) Staining of the isolated organellar fraction with MitoTracker Red, (C) Localization of PfTPx ${ }_{G \mid}$ to the membranes of intact/permeabilized apicoplasts from control parasites, (D) Absence of PfTPx ${ }_{G 1}$ in the membranes of intact/permeabilized apicoplasts from $\mathrm{AlF}_{4}$ treated parasites. Scale Bars as indicated in the figures. (E) Thermolysin treatment of isolated organelles demonstrates outermost membrane localization of PfTPx $\mathrm{Gl}_{\text {. }}$ 
(A)

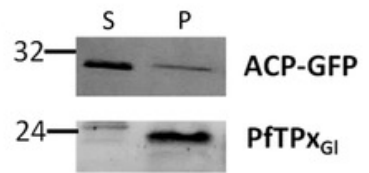

(B)

Mitotracker Red

ACP-GFP

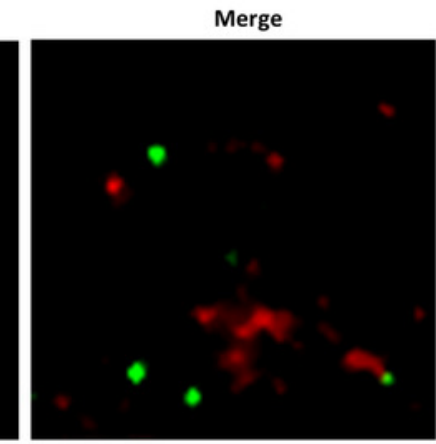

(C) Intact Organelles

Permeabillized Organelles
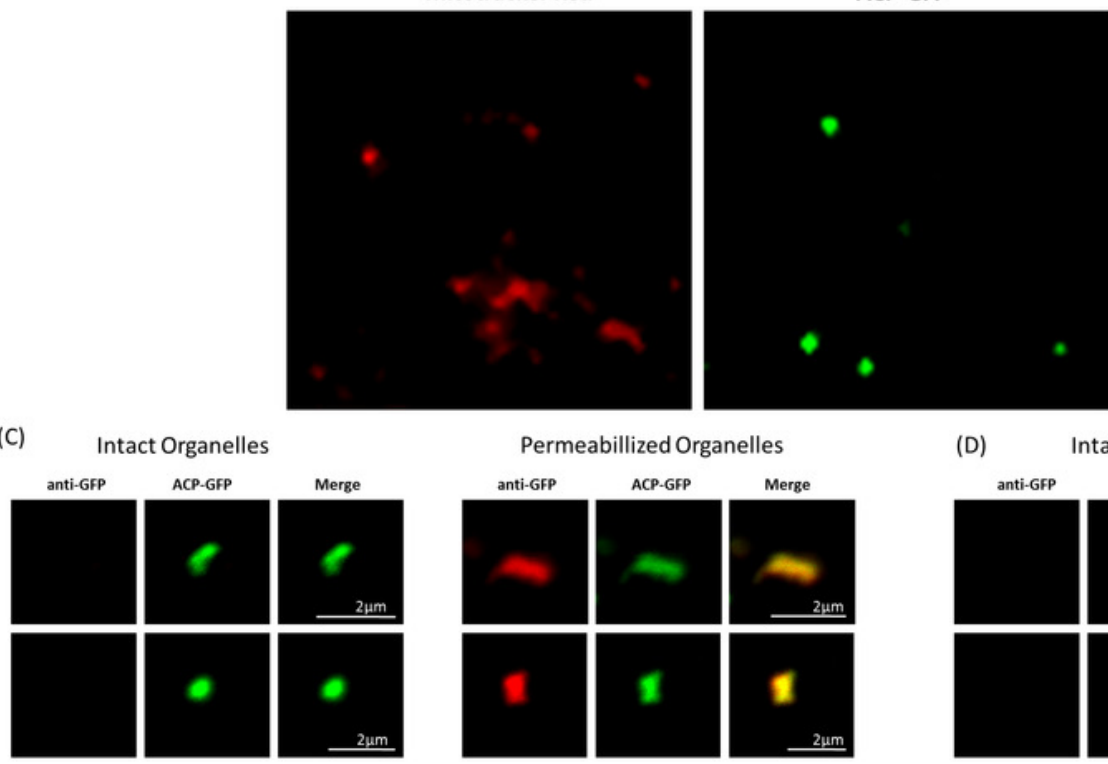

(D) Intact Organelles
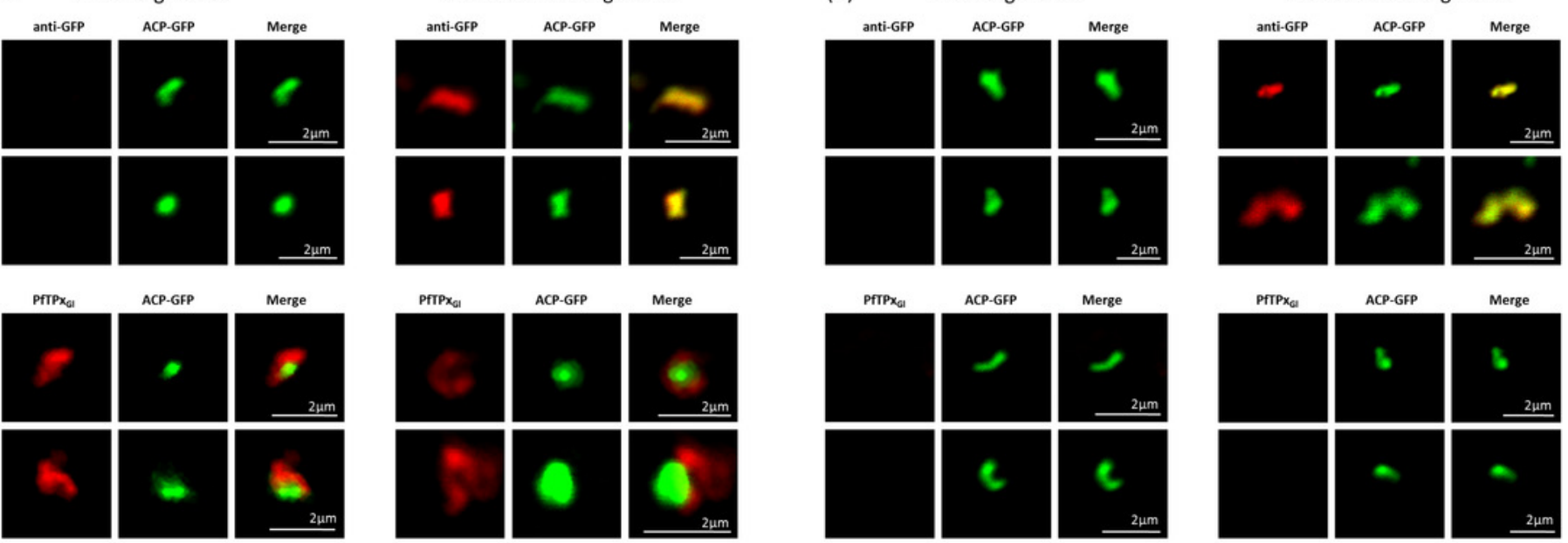

(E)

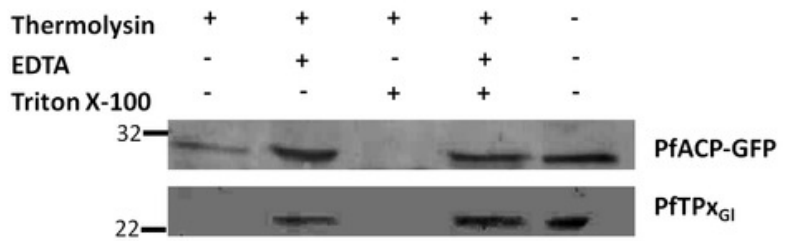


Figure 7

Schematic representation of secretory protein targeting pathways in Plasmodium falciparum.

Arrows indicate the direction of the secretory protein traffic.

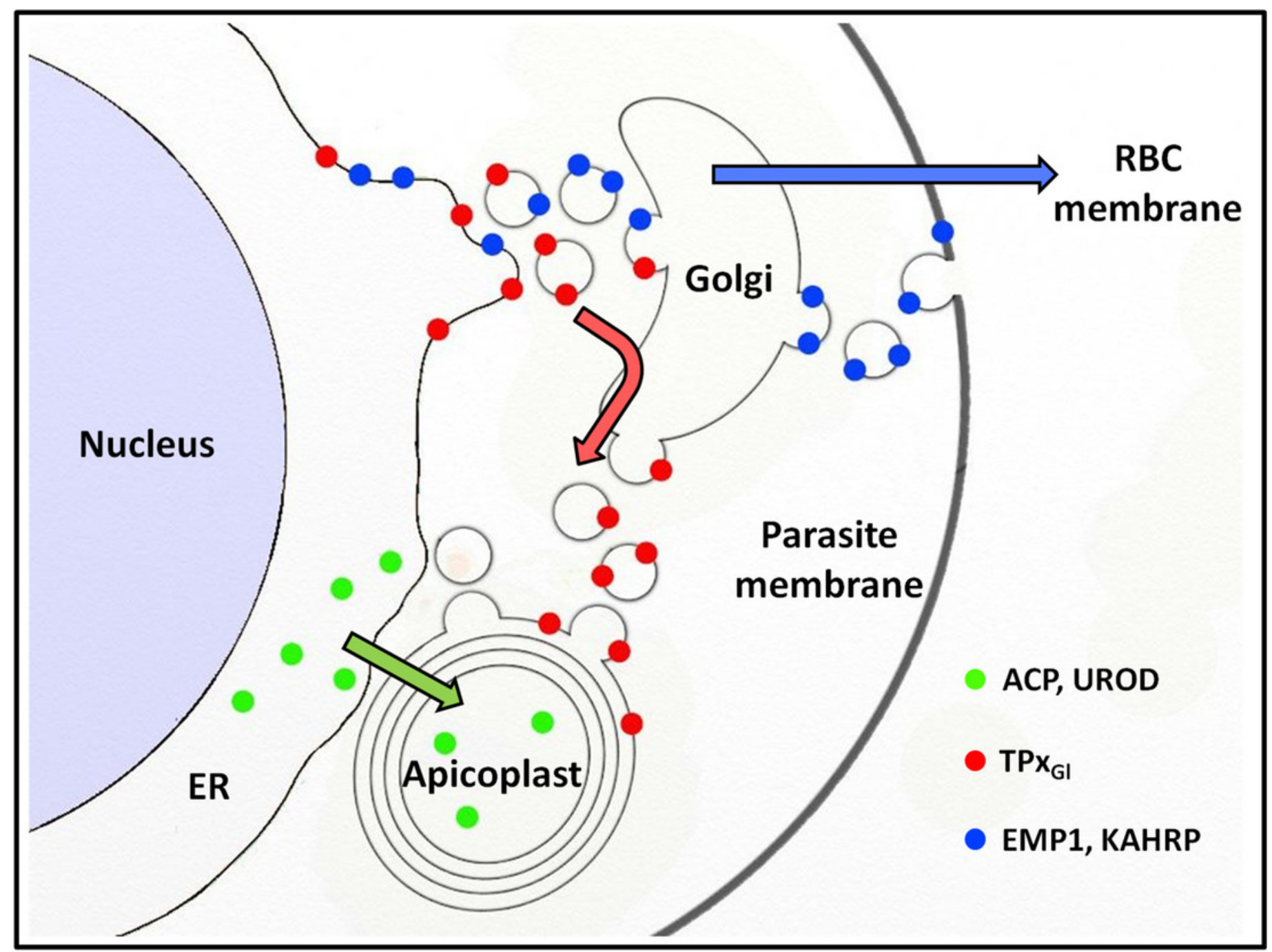

